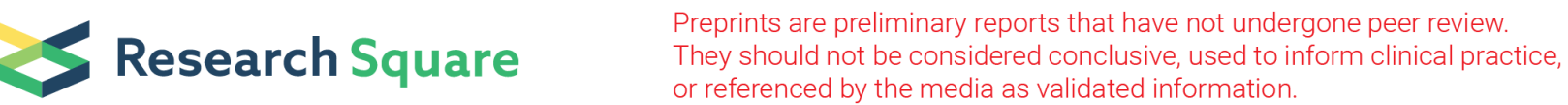

\section{FGF20 protected against BBB disruption after traumatic brain injury by activating the AKT/GSK3 $\beta$ pathway to upregulate junction protein expression and inhibiting JNK/NFKB-dependent neuroinflammation}

\author{
Jun Chen
}

Wenzhou Medical University

Xue Wang

Wenzhou Medical University

Jian Hu

Wenzhou Medical University

Wenting Huang

Wenzhou Medical University

Confidence Dordoe

Wenzhou Medical University

Kaiming Guo

Wenzhou Medical University

Fanyi Han

Wenzhou Medical University

Ruili Guo

Wenzhou Medical University

Shasha Ye

Wenzhou Medical University

Jingting Du

Wenzhou Medical University

Li Lin ( $\nabla$ linliwz@163.com )

Wenzhou Medical University https://orcid.org/0000-0003-3355-6139

Xiaokun Li

Wenzhou Medical University

Research

Keywords: traumatic brain injury, blood-brain barrier, tight junction protein, inflammatory response, FGF20 
Posted Date: December 23rd, 2019

DOl: https://doi.org/10.21203/rs.2.19529/v1

License: (c) (i) This work is licensed under a Creative Commons Attribution 4.0 International License. Read Full License 


\section{Abstract}

Background $\triangle \mathrm{B}$ lood-brain barrier (BBB) disruption and the cerebral inflammatory response are two reciprocal mechanisms that work together to mediate the degree of brain edema, which is responsible for the majority of deaths after traumatic brain injury (TBI), and facilitate further brain damage, which leads to long-term TBI complications. Fibroblast growth factor 20 (FGF20), a neurotrophic factor, plays important roles in the development of dopaminergic neurons in Parkinson disease (PD). However, little is known about the role of FGF20 in TBI. The aim of the current study was to assess the protective effects of FGF20 in TBI through protecting the BBB.

Methods: We explored the relationship between FGF20 and BBB function in controlled cortical impact (CCI)-induced TBI mice model and TNF-a-induced human brain microvascular endothelial cell (HBMEC) in vitro BBB disruption model. We also explored the mechanisms of these interactions and the signaling processes involved in BBB function and neuroinflammation.

Results: In this study, we demonstrate that recombinant human FGF20 (rhFGF20) reduced neurofunctional deficits, brain edema and Evans Blue penetration in vivo after TBI. In an in vitro BBB disruption model of, rhFGF20 could reverse changes to TNF-a-induced HBMEC morphology, reduce Transwell permeability, and increase transendothelial electrical resistance (TEER). In both a TBI mouse model and in vitro, rhFGF20 upregulated the expression of BBB-associated tight junction (TJ) protein and adherens junction (AJ) protein via the AKT/GSK3 $\beta$ pathway. In addition, rhFGF20 inhibited the cerebral inflammatory response through regulating the JNK/NFKB pathway and further protected the function of the BBB.

Conclusions : Our results contribute to a new treatment strategy in TBI research. FGF20 is a potential candidate to treat TBI as it protects the BBB via regulating the AKT/GSK3 $\beta$ and JNK/NFKB signaling pathways.

\section{Background}

Traumatic brain injury (TBI) is a leading cause of death and disability worldwide, especially in children and young adults $[1,2]$. TBI-induced brain damage comprises two pathological phases. Primary damage occurs at the moment of trauma [3], while secondary damage, including excitotoxicity, blood-brain barrier (BBB) disruption, oxidative stress, neuroinflammation, ischemia, edema, oxidative stress and so forth [4], is often related to cognitive and behavioral dysfunction[5]. Among these pathological events, BBB disruption and cerebral inflammation are two reciprocal mechanisms that are vital to the progression of brain injury and long-term neurological deficits [6].

The BBB plays an important role in separating pools of neurotransmitters and neuroactive agents to regulate the brain microenvironment [7]. Accumulating studies have indicated that brain edema and intracranial hypertension caused by BBB disruption after TBI have become a major cause of death [7]. The main constituent of the BBB is cerebrovascular endothelial cells (ECs), which are connected to a 
whole vascular system by consecutive intercellular tight junctions (TJ) protein and adherence junction (AJ) protein [8]. TJ and AJ protein limit paracellular diffusion between ECs, leading to the high transendothelial electrical resistance (TEER) of the BBB [9]. The localization and expression of TJs and AJs profoundly influence the integrity of the BBB. The expression levels of claudin-5, Occludin, zonula occludens-1 (ZO-1) and VE-Cadherin are generally detected to evaluate the loss of BBB integrity [10].

The inflammatory reaction to TBI occurs through peripheral immune mediators at the entrance to the central nervous system (CNS) via a damaged BBB [11]. Posttraumatic inflammation can be harmful by mediating neuronal death and promoting neurodegeneration [12]. In particular, previous studies have demonstrated that tumor necrosis factor- $a$ (TNF-a), interleukin (IL)-1 $1 \beta$, IL- 6 and iNOS are crucial proinflammatory cytokines following trauma [13-15]. Interestingly, a number of studies have shown that inflammatory challenge aggravates and increases BBB permeability [16]. Therefore, a drug targeting BBB integrity and the inflammatory response is a potential therapeutic strategy for TBI.

Fibroblast growth factor 20 (FGF20) is a paracrine growth factor and member of the fibroblast growth factor (FGF) family. It was originally identified as a new FGF preferentially expressed in the substantia nigra pars compacta (SNpc). A previous study demonstrated that FGF20 is predominantly expressed in the brain, with little or even no FGF20 expression in other organs in adults[17]. This expression profile has prompted scientists to research the relationship between FGF20 and the brain. Accumulating evidence on FGF20 has shown its well-known neurotrophic activity in cultured neurons [18], and FGF20 has been suggested to play an important role in regulating CNS development and function [19]. FGF20 showed neurotrophic activity in cultured dopaminergic neurons and Parkinson disease (PD) [20]. In addition, FGF20 mutation may induce PD, indicating that FGF20 is involved in the etiology and therapy of PD.

The positive roles of FGF20 in PD imply that it may play a neuroprotective role in other CNS disorders. However, the effects of FGF20 and its possible underlying mechanism of action on BBB integrity following TBI remain unknown. In the present study, we first aimed to determine the therapeutic effect of recombined human FGF20 (rhFGF20) after TBI both in vivo and in vitro. We evaluated whether rhFGF20 can ameliorate the pathophysiological symptoms of acute TBI by preserving BBB integrity and reducing the inflammatory response. We further studied whether rhFGF20 protects BBB integrity via the $A K T / G S K 3 \beta$ pathway and inhibits the inflammatory response through regulating the JNK/NFKB pathway. Taken together, our results contribute to the development of new treatment strategies for rhFGF20 in TBI research.

\section{Materials And Methods}

\section{Reagents and antibodies}

The following reagents including AKT inhibitor LY294002, GSK3 $\beta$ inhibitor SB216763, JNK agonist Anisomycin, NFKB inhibitor Bay 11-7082, FITC-dextran, and Evans Blue were purchased from Sigma (Sigma-Aldrich, St Louis, MO). TNF-a was obtained from R\&D Systems (Minneapolis, MN, USA). The primary antibodies applied in this study were as follows: anti-p-GSK3 3 (No. 9336S), anti-GSK3 $\beta$ (No. 
9832S), anti-p-JNK (No. 4668S), anti-JNK (No. 9252S), anti-p-NFkB p65 (No. 3033S) and anti-NFkB (No. 8242S) antibodies were purchased from Cell Signaling Technology (Danvers, MA, USA); anti-FGF20 (No. ab198876), anti-VE-cadherin (No. ab33168), and anti-claudin-5 (No. ab131259) antibodies were obtained from Abcam (Cambridge, MA, USA); anti-p-AKT (No. sc-7985-R), anti-AKT (No. sc-8312) and anti-occludin (No. 71-1500) antibody were parchsed from Invitrogen (Carlsbad, CA, USA) and Santa Cruz Biotechnology (Dallas, TX, UAS), respectively. The secondary antibodies used in this study were goat anti-rabbit immunoglobulin G (IgG) H\&L (HRP) (No. ab6721) and goat anti-mouse IgG-HRP (No. ab6278) were purchased from Abcam (Cambridge, MA, USA) and Santa Cruz Biotechnology (Dallas, TX, UAS), respectively.

\section{Animals and surgical procedures used to develop a controlled cortical impact (CCl)-induced TBI model}

C57BL/6 male mice (8-12 weeks, 22-28 g) purchased from the Animal Center of the Chinese Academy of Sciences (Beijing, China) were used in this study. The animal protocols were approved by the Animal Center of the Chinese Academy of Science (Shanghai, China) and the Laboratory Animal Ethics Committee of Wenzhou Medical University.

The surgical procedures to construct the controlled cortical impact (CCI)-induced TBI model were performed as previously described [21, 22]. Briefly, male C57BL/6N mice were anesthetized with $4 \%$ chloral hydrate ( $10 \mathrm{~mL} / \mathrm{kg}$, ip), administered normal saline or $0.5 \mathrm{mg} / \mathrm{kg}$ rhFGF20 by nasal delivery, and mounted on a stereotaxic frame. A $4 \mathrm{~mm}$ craniotomy was performed on the left parietal bone midway between the bregma and lambda sutures with the medial edge $0.55 \mathrm{~mm}$ lateral to the midline. The skullcap was carefully removed, and the $\mathrm{CCl}$ injury was induced with a $3 \mathrm{~mm}$ diameter impactor at 3.0 $\mathrm{m} / \mathrm{s}$ at a depth of $1.0 \mathrm{~mm}$. After the injury, the skin was sutured, and the temperature of the mouse was maintained at $37^{\circ} \mathrm{C}$. Mice in the sham group underwent the craniotomy procedure but were not impacted (Figure 1A).

\section{Rotarod test}

The rotarod test protocol was described as previously [23].Briefly, mice underwent a 3-day training phase with a rotarod (IITC Life Science) that was gradually accelerated from 5 to $30 \mathrm{rpm}$ over $5 \mathrm{~min}$. During the procedure, the rotarod latency was recorded as the time before the mouse fell off the rod equipment or gripped the rod for two successive revolutions after TBI.

\section{Neurobehavioral assessment}

Neurobehavioral function was evaluated with the Garcia test as described previously [24]. The Garcia test was conducted in mice $24 \mathrm{~h}$ after surgery under blinded conditions. Sensorimotor deficits were assessed by a modified Garcia test that included assessments of spontaneous activity, symmetry in the movement of four limbs, forepaw outstretching, climbing, body proprioception and response to vibrissae touch and scored from 0 to 18, with a score of 0 to 3 awarded for each test.

\section{Grip strength test}


The grip strength test was performed as described in previous research [25]. Each mouse was placed over a grid used to measure forelimb strength (San Diego Instruments, San Diego, CA, USA) by the tail such that its forepaws were allowed to grasp the grid. The mouse was then pulled backward until its released its grip. The baseline grip strength of each animal was measured one day before $\mathrm{CCl}$ or the sham procedure.

\section{Evans blue extravasation}

BBB permeability was investigated on the 24 hours after $\mathrm{CCl}$ injury by monitoring extravasation of Evans blue dye. The assessment was performed as described previously [26]. The $0.25 \mathrm{~mL}$ EB dye (2\% in saline) was injected intravenously at 4 hours before sacrificing of mice to visualize the BBB leakage. The anesthetized animals were transcardially perfused with saline to sufficiently wash away the intravascular-localized dye. The left hemisphere was immediately weighed and homogenate in 5 times volume formamide. The homogenate was incubated for 3 days in $72{ }^{\circ} \mathrm{C}$ and was centrifuged $(15000$ rpm, $30 \mathrm{~min}$ ). The supernatant's absorbance was detected by spectrophotometer (Bio- Rad, Hercules, CA, USA) at an excitation wavelength of $610 \mathrm{~nm}$ and an emission wavelength of $680 \mathrm{~nm}$.

\section{Brain water content}

Brain edema was evaluated by the water content in brain tissue using the wet-dry weight method at 24 hours after TBI, as previously reported [21]. Brain tissue was obtained without transcardiac perfusion. After removal, the brain tissue was immediately weighed to determine as wet weight. Subsequently, the brain was dried in an oven $\left(100^{\circ} \mathrm{C}\right)$ for 24 hours to determine as dry weight by the same electronic balance. Brain water content (\%) was calculated as: [ (wet brain weight -dry brain weight)/wet brain weight] $\times 100 \%$

\section{Western blot}

Western bolt analysis was performed to detect the expression of BBB-associated TJ and AJ proteins, as well as proteins involved in the signaling. At 24 hours following $\mathrm{CCl}$, brain tissue proteins were extracted from $5 \mathrm{~mm}$ brain coronal sections upon the site of impact. The cell total proteins and membrane protein were extracted after TNF-a treating 24 hours. An equivalent amount of protein $(80 \mu \mathrm{g}$ for in vivo and 60 $\mu \mathrm{g}$ for in vitro) was separated by SDS-PAGE gel, and then transferred to PVDF membrane. Membrane was blocked by $5 \%$ nonfat milk in TBST for 90 minutes at room temperature. And then incubated with primary antibodies: ZO-1 (1:500), VE-Cadherin (1:1000), Occludin (1:1000), Claudin-5 (1:800,), p-Akt (1:1000), Akt (1:1000), p-GSK3 3 (1:1000), GSK3 3 (1:1000), p-NFKB (1:1000), NFKB (1:1000) at $4{ }^{\circ} \mathrm{C}$ overnight. The membrane was washed three times in TBST and then covered with respective secondary antibodies for 1 hour at room temperature. Finally, membrane was incubated with enhanced chemiluminescence (ECL), then imaged and quantified by Image Lab 3.0 software (Bio- Rad, Hercules, CA, USA). 
For in vivo protein evaluation, animals were anesthetized by $4 \%$ chloralic hydras $(0.2 \mathrm{~mL} / \mathrm{mice}$, i.p.) at 24 hours following TBI. The whole brain was post-fixed by $4 \%$ paraformaldehyde for $12 \mathrm{~h}$ and embedded in paraffin. Transverse sections ( $5 \mu \mathrm{m}$ thick) were mounted on slides that are for subsequent staining. Hematoxylin \& eosin (H\&E) and Nissl staining were performed to observe histopathological changes based on the manufacture's protocol. Images were acquired using a light microscopy.

Immunofluorescence staining was applied to detect the protein expression. For vivo protein detection, sections were deparaffinized and rehydrated. Then tissue was incubated with $3 \% \mathrm{H}_{2} \mathrm{O}_{2}$ (dilute in methanol) for $15 \mathrm{~min}$, followed by blocked nonspecific binding in $5 \%$ bovine serum albumin (BSA) for 30 $\min$ at $37^{\circ} \mathrm{C}$. Then sections were treated with primary antibodies overnight at $4^{\circ} \mathrm{C}$. The following primary antibodies were applied: ZO-1 (1:300), VE-Cadherin (1:300), Occludin (1:300), Claudin-5 (1:300), and Iba-1 (1:100). The sections were washed with PBST three times for $5 \mathrm{~min}$ and incubated with AlexaFluor 488 or AlexaFluor 647 donkey anti-rabbit/goat secondary antibodies $(1: 1000)$ for $1 \mathrm{~h}$ at $37^{\circ} \mathrm{C}$. Then the nuclei were stained with DAPI for 5 min. For in vitro protein evaluation, HBMECs were plated in 12-well plate. The cells were washed with PBS, and fixed with $4 \%$ paraformaldehyde in PBS for 30 min in room temperature. Then, cells were blocked with 5\% BSA in PBS containing $0.1 \%$ Triton X-100 for 30 min in $37^{\circ} \mathrm{C}$. The next process as described previously in tissue section. The stained sections were stored at $4{ }^{\circ} \mathrm{C}$ and imaged at $\times 400$ magnifications a Nikon ECLPSE 80i fluorescence microscope (Niko, Japan).

\section{RNA extraction and RT-PCR}

Total RNA was extracted from $5 \mathrm{~mm}$ brain coronal sections upon the site of impact or HBMECs by TriPure Isolation Reagent (Roche, South San Francisco, CA, USA). The RNA was quantified by Nanodrop spectrometry (Thermo Fisher Scientific, MA, USA) and the sample with the OD 260/280 ratio between 1.52.0 was used. $1 \mu \mathrm{g}$ of RNA was used to synthesize cDNA by PrimeScript RT Reagent Kit (RR037A, TaKaRa, Japan). Reverse transcription was conducted with SYBR Green PCR Master Mix (Bio- Rad, Hercules, CA, USA) in $10 \mu \mathrm{L}$ final reaction volume. The PCR primer pairs are listed in Table 1, purchased from Sangon Biotech (Shanghai, China). The cycling program was an initial hold at $95^{\circ} \mathrm{C}$ for $5 \mathrm{~min}$, followed by 40 cycles of denaturation at $95^{\circ} \mathrm{C}$ for $30 \mathrm{~s}$, annealing at $62^{\circ} \mathrm{C}$ for $30 \mathrm{~s}$, and extension at 72 ${ }^{\circ} \mathrm{C}$ for $30 \mathrm{~s}$. The mRNA expression levels of the target genes were normalized to the mRNA expression level of $\beta$-actin, and calculated by the $2^{-\triangle \Delta C T}$ method. The results are expressed as the means $\pm \mathrm{SEMs}$ of duplicate samples from three independent experiments.

\section{Cell culture and in vitro BBB disruption model}

Primary HBMECs (ACBRI376, Cell Systems Corporation, Kirkland, WA) were grown in EBM-2 supplemented with $10 \%$ fetal bovine serum (FBS) and $1 \%$ penicillin-streptomycin in cell culture plates coated with fibronectin. The plates were incubated at $37^{\circ} \mathrm{C}$ in a humidified atmosphere of $5 \% \mathrm{CO}_{2}$. Recombinant human TNF-a, as a well-established cytokine mediator of vascular leakage, was used to construct the BBB disruption model as described previously [27]. Briefly, cells that had reached approximately $90 \%$ confluence were treated with TNF-a $(50 \mathrm{ng} / \mathrm{mL})$ with or without rhFGF20 $(50 \mathrm{nM})$ for 
$24 \mathrm{~h}$. To further illustrate the effect of TNF-a treatment, the cells were treated with the AKT inhibitor LY294002 $(20 \mu \mathrm{M})$ [26], the GSK3 $\beta$ inhibitor SB216763 (30 $\mu \mathrm{M})$ [28], the JNK agonist anisomycin $(4 \mu \mathrm{M})$ [29], or the NFKB inhibitor Bay 11-7082 (5 $\mu \mathrm{M})$ [30] for $24 \mathrm{~h}$ and then harvested for further analysis.

\section{FITC-dextran extravasation}

FITC-dextran extravasation assays were performed to quantification endothelial permeability of HBMECs as previously described [31]. In brief, HBMECs were cultured at $1 \times 10^{5}$ cells/well on polycarbonate 24well transwell insert chambers with $0.4-\mathrm{mm}$ pores. After 2 days, cells were proliferated until compact density and then were administrated with or without TNF- $a$ and rhFGF20. After $24 \mathrm{~h}$, the medium of transwell insert chambers was replaced new medium containing $1 \%$ FITC-dextran ( $10 \mathrm{mg} / \mathrm{mL})$. After $4 \mathrm{~h}$, the fluorescence of each well was detected with an EnSpire Manager (PerkinElmer Company) multimode microplate reader, the excitation wavelength is $485 \mathrm{~nm}$ and emission wavelengths is $520 \mathrm{~nm}$.

\section{Trans-endothelial electrical resistance (TEER)}

TEER indicating human brain microvascular endothelial (HBMEC) monolayer permeability was detected with an EndOhm- 6 chamber and EVOM resistance meter (World Precision Instruments, Sarasota, FL) as previously described [31]. In brief, a 6-mm Transwell insert chamber with cultured HBMECs was transferred into an EndOhm- 6 chamber with $0.1 \mathrm{M} \mathrm{KCl}$. Then, the culture medium in the Transwell insert chamber was replaced with $0.1 \mathrm{M} \mathrm{KCl}$. The EndOhm cap was inserted on the top chamber, and the top chamber and Transwell insert chamber were connected though a connector cable. Resistance was then measured with an EVOM resistance meter. A blank Transwell chamber without HBMECs was used as a blank control.

\section{Statistical analysis}

GraphPad Prism software (GraphPad Software, Inc., San Diego, CA) was used for the data analyses. The results are expressed as the mean \pm standard error of the mean (SEM). Statistical differences among data from three and moree groups were evaluated with one-way analysis of variance (ANOVA) followed by Tukey-Kramer's test. $P<0.05$ was considered statistically significant.

\section{Results}

\section{rhFGF20 treatment improved neurofunctional and behavioral recovery following TBI in mice}

To assess the correlation of FGF20 expression with TBI, we investigated the protein expression levels of FGF20 in mouse brains. The expression level of FGF20 in the TBI group was significantly reduced compared with the sham group, and the intranasal delivery of FGF20 increased the FGF20 level in the brain after TBI (Figure 1B and 1C). These results indicated that FGF20 expression is correlated with TBI.

Then, we investigated whether rhFGF20 promotes neurofunctional and behavioral recovery following TBI. The Garcia test was used to monitor neurofunctional deficits in the TBI mice [24]. The results of the 
Garcia test showed that mice in the TBI group exhibited neurofunctional behavior deficits after TBI and that rhFGF20 administration markedly ameliorated these neurofunctional deficits (Figure 1D). The grip strength test was used to assess forelimb function [25]. As shown in Figure 1E, mice in the TBI group exhibited functional deficits in forelimb strength; however, the administration of rhFGF20 diminished these functional deficits. The rotarod test measures motor coordination in mice. Rotarod latency measured in the rotarod test was decreased in mice in the TBI group, whereas rhFGF20 clearly increased the latency (Figure 1F). Brain water content analysis is used to measure the degree of cerebral edema, an important predictor of TBI prognosis. The brain water content was significantly decreased in mice in the rhFGF20 treatment group compared to mice in the TBI group $24 \mathrm{~h}$ after TBI (Figure 1G). All of the above results demonstrate that endogenous FGF20 expression was reduced after TBI and that exogenous FGF20 ameliorated functional behavior deficits and decreased the degree of cerebral edema after TBI.

Furthermore, hematoxylin and eosin (H\&E) and Nissl staining of brain sections was performed to assess the effect of rhFGF20 on brain tissue and neuron status. H\&E staining showed that mice in the TBI group exhibited remarkable brain tissue damage in the cortex. However, rhFGF20 protected against brain tissue damage after TBI (Figure 2A-B). In addition, Nissl staining showed that rhFGF20 decreased neuronal loss after TBI (Figure 2C-D).

\section{rhFGF20 ameliorated TBI-induced BBB disruption by increasing the expression of junction proteins}

In an Evans Blue extravasation experiment, which can be used to indicate BBB disruption, Evans Blue dye leaked though the BBB and remained in the cortex [23]. Compared with the sham group, Evans Blue dye extravasation through the BBB was significantly increased in the TBI group, while treatment with rhFGF20 dramatically reduced leakage (Figure 3A-B), which indicated that rhFGF20 could ameliorate TBIinduced BBB damage.

TJ and AJ proteins are important mediators of BBB integrity. To elucidate the effect of rhFGF20 on TJ and AJ proteins, we detected junction protein levels after TBI by western blotting and immunofluorescence analysis. Western blotting showed that TBI significantly downregulated the expression of VE-Cadherin, Occludin and Claudin-5. In contrast, rhFGF20 markedly upregulated the expression of these proteins (Figure 3C-F). The results of immunofluorescence analysis were consistent with those of western blotting. These results confirmed that VE-Cadherin, Occludin and Claudin- 5 were significantly increased after rhFGF20 treatment compared with their levels in the TBI group (Figure 3G). Based on these data, we determined that rhFGF20 treatment protected against BBB disruption after TBI at least in part by upregulating the expression of TJ and AJ proteins.

\section{rhFGF20 inhibited neuroinflammation by suppressing microglial activation and the inflammatory response after TBI}

TBI induces neuroinflammation response that increases several inflammatory factors, producing further damage. To illustrate the anti-inflammatory effect of rhFGF20 after TBI, we detected the degree of microglial activation and expression levels of the proinflammatory cytokines IL-1 $\beta$, IL-6 and iNOS. 
Microglial activation, a key manifestation of CNS neuroinflammation[32], was assessed by immunofluorescence labeling to determine the cell number, soma area, and process length. The results showed that TBI clearly increased the number of Iba1-positive microglia in the cortex and activated microglia, as indicated by the increased soma area and shorter ramified processes compared with the sham group. However, rhFGF20 treatment inhibited neuroinflammation by reducing the number of activated microglia and abnormal phenotypic changes (Figure 4A-D).

The expression levels of the critical proinflammatory cytokines IL-1 $\beta$, IL-6, and iNOS were measured by RT-PCR. Subjection to TBI obviously increased the production of IL-1 $\beta$, IL-6 and iNOS in the brain tissue. However, rhFGF20 treatment significantly reduced the expression of these proinflammatory cytokines (Figure 4E-G). Based on the above data, we believe that rhFGF20 treatment can inhibit neuroinflammation and protect against BBB disruption following TBI by suppressing the inflammatory response.

\section{rhFGF20 improved recovery after TBI via the AKT/GSK3 $\beta$ and JNK/NFKB signaling pathways}

In the current study, to illustrate the mechanism of the protective effect of rhFGF20 after TBI, we detected downstream pathway proteins. Previous studies have shown that TBI-induced cell apoptosis is correlated with AKT [33]. Consistent with previous results, our results showed that the ratio of phosphorylated AKT to total AKT was markedly decreased in TBI mice; however, rhFGF20 administration significantly rescued the TBI-induced decrease in the phosphorylation of AKT (Figure 5A and 5B). As shown by a previous study, AKT phosphorylation induced a decrease in GSK3 activity by increasing the phosphorylation of GSK3ß [34]. GSK3 deactivation was shown to increase the expression of neuroprotective and neurotrophic proteins [35] and regulate TJ protein expression[36]. To further assess whether the protective effects of rnFGF20 against BBB disruption were mediated through GSK3 $\beta$ inhibition, the levels of total GSK3 $\beta$ and phosphorylated GSK3 $\beta$ were determined. The ratio of phosphorylated GSK3 $\beta$ to total GSK3 $\beta$ was significantly decreased after TBI, but rhFGF20 increased the level of GSK3 $\beta$ phosphorylation (Figure $5 \mathrm{~A}$ and $5 \mathrm{C})$.

Inflammation is a key driving factor in the pathogenesis of TBI. As a major component of the MAPK signaling pathway, JNK plays an important role in inflammatory disease [37]. In addition, the transcription factor NFKB is a major regulator of inflammation. We detected $J N K$ and NFKB, inflammatory pathway proteins, in our study. As shown in Figure 5D and 5E, the level of p-JNK was increased after TBl; however, rhFGF20 decreased the level of p-JNK after TBI. Furthermore, we detected the level of p-NFKB p65 in the total protein to show the degree of NFKB nuclear translocation and activity. The expression level of p-NFKB p65 was significantly increased in the TBI group, whereas rhFGF20 treatment reduced TBI-induced NFKB p65 phosphorylation (Figure 5D and 5F).

\section{rhFGF20 protected against TNF-a-induced BBB injury}

To further confirm the hypothesis that rhFGF20 protects against TNFa-induced BBB damage in a cellular model, HBMECs were treated with TNF-a $(50 \mathrm{ng} / \mathrm{mL})$, followed by treatment with $50 \mathrm{nM}$ rhFGF20. 
The morphology of TNF-a-induced HBMECs after rhFGF20 treatment was observed. TNF-a-treated cells were more slender in shape and exhibited a larger paracellular space compared to the control group; however, rhFGF20 rescued these change in cell morphology (Figure 6A). Furthermore, we detected the proliferative ability of cells treated with rhFGF20. The results suggested that TNF-a and rhFGF20 did not promote cell proliferation (Figure 6B). Therefore, the protective effect of rhFGF20 against the TNF-ainduced disruption of endothelial monolayer permeability was not through increased cell viability. A FITCdextran extravasation experiment was used to illustrate the paracellular permeability of ECs. Our results showed that the paracellular permeability of HBMECs significantly increased after TNF-a treatment (Figure 6C). Thus, we believe that the TNF-a-induced cellular model of BBB disruption in our research is feasible. In addition, rhFGF20 treatment clearly reduced FITC-dextran permeation (Figure 6C). EC resistance is also an important indicator of paracellular permeability, as a high resistance indicates low paracellular permeability and a high degree of barrier integrity. Consistent with the results of the FITCdextran extravasation experiment, determination of the TEER showed that TNF-a decreased EC resistance, whereas rhFGF20 treatment ameliorated this decrease in resistance (Figure 6D). Our results illustrated that rhFGF20 treatment ameliorated TNF-a-induced endothelial permeability.

The expression of inflammatory factors (IL-1 $1 \beta$, IL-6 and iNOS) was also assessed in this in vitro BBB disruption model. The results showed that the expression of inflammatory factors (IL-1 $\beta$, IL- 6 and iNOS) was significantly increased in TNF-a-induced HBMECs; however, rhFGF20 had an anti-inflammatory effect on TNFa-induced HBMECs by decreasing the level of these inflammatory factors (Figure 6E-G).

\section{rhFGF20 protected against TNF-a-induced BBB injury by increasing junction protein expression}

TJ and AJ proteins are important regulators of paracellular permeability in ECs. Therefore, we hypothesized that the TNF-a-induced modulation of TJ and AJ proteins would increase the paracellular permeability of HBMECs. TNFa significantly decreased the levels of VE-cadherin, occludin and claudin-5 in the total protein; however, rhFGF20 increased the levels of these proteins (Figure 7A-D). These junction proteins are known to be distributed mainly in the cellular membrane; we therefore measured their expression levels in the cell membrane. Consistent with previous findings, our data showed that TNF-a markedly decreased the levels of VE-Cadherin, occludin and claudin-5 in the cellular membrane; however, rhFGF20 rescued changes to these protein levels (Figure 7E-H).

\section{rhFGF20 protected against TNF- $a-i n d u c e d ~ B B B$ injury via the AKT/GSK3 $\beta$ signaling pathway}

To evaluate whether the effects of rhFGF20 on TNF-a-induced BBB injury occurred through the $A K T / G S K 3 \beta$ pathways and determine whether the AKT/GSK3 $\beta$ pathway is involved in preservation of the $\mathrm{BBB}$ in vitro, HBMECs under TNF-a conditions were treated with rhFGF20. We detected the expression level of p-AKT and compared it to the total AKT level. As shown in Figure $8 \mathrm{~A}$ and $8 \mathrm{~B}$, consistent with the in vivo results, rhFGF20 significantly rescued the TNF-a-induced decrease in AKT phosphorylation. To determine whether the protective effect of rhFGF20 on the BBB observed in our study is related to AKT, the AKT inhibitor LY294002 was used for cotreatment with rhFGF20. We found that LY294002 not only significantly reversed the restorative effect of rhFGF20 on BBB permeability and endothelial resistance 
(Figure $8 \mathrm{C}$ and $8 \mathrm{D}$ ) but also decreased the expression of Claudin-5, a critical determinant of the integrity of interendothelial junctions within the BBB [38] (Fig 8E and 8F). We further assessed the effect of rhFGF20 downstream of AKT and found that LY294002 significantly inhibited the rhFGF20-induced increase in the level of p-GSK3 $\beta$ (Figure 8G and 8H). The GSK3 $\beta$ inhibitor SB216763 was applied to examine the role of GSK3 $\beta$ in the protective effect of rhFGF20 on endothelial monolayer permeability. SB216763 also reversed the protective effect of rhFGF20 on permeability and endothelial resistance (Figure $8 \mathrm{I}$ and $8 \mathrm{~J}$ ). These results further confirmed that rhFGF20 protects against TNF-a-induced BBB injury via the AKT/GSK3 $\beta$ signaling pathway.

\section{rhFGF20 protected against TNF-a-induced BBB injury by inhibiting the inflammatory response via the JNK/NFKB signaling pathway}

In addition, we investigated the anti-inflammatory mechanism by which rhFGF20 protects against BBB injury. The JNK signaling pathway is important for endothelial inflammation [39]. To test whether the protective effect of rhFGF20 on the BBB is related to JNK and inflammation, we detected the expression level of p-JNK and compared it to that of JNK. As shown in Figure 9A and 9B, consistent with the in vivo results, rhFGF20 significantly decreased the TNF-a-induced increase in JNK phosphorylation. Furthermore, we used the JNK agonist anisomycin for cotreatment with rhFGF20 and detected the expression level of $\mathrm{p}$-JNK for comparison to the total JNK level. Anisomycin not only significantly reversed the restorative effect of rhFGF20 on BBB permeability and endothelial resistance (Figure 9C-D) but also increased IL-1 $\beta$, IL-6 and iNOS mRNA expression (Figure 9E-G). Many downstream targets in the JNK signaling pathway include various transcription elements. The transcription factor NFKB, a major regulator of inflammation, regulates the transcription of a vast number of proinflammatory genes and plays a fundamental role in innate immune and inflammation. We speculated that NFKB is a downstream factor of the JNK pathway. We further assessed the anti-inflammatory effects of rhFGF20 downstream of JNK and found that anisomycin significantly inhibited the rhFGF20-induced decrease in NFKB p65 nuclear translocation detected by both western blotting and immunofluorescence analysis (Figure 10A-C). The NFKB inhibitor Bay 11-7082 was applied to examine the effect of NFKB on endothelial monolayer permeability. Bay 11-7082 also reversed TNFa-induced injury to BBB permeability and endothelial resistance (Figure 10D and 10E). These results further confirmed that rhFGF20 protects against TNF-ainduced BBB injury by decreasing the inflammatory response via inhibiting the JNK/NFKB signaling pathway.

\section{Discussion}

TBI is associated with a range of postinjury complaints, and there are currently no fully effective therapies for most of these symptoms $[3,40,41]$. The focus on TBI research has primarily been cognitive and/or motor symptoms, and less attention has been paid to the neuropsychiatric symptoms frequently identified in patients after TBI $[42,43]$. The $\mathrm{CCI}$ model is a stable and well-established animal model used to research TBI since 1988 [44]. Various parameters (velocity, depth, and dwell time) can be selected for $\mathrm{CCl}$ model development to achieve the required degree of injury with great reproducibility [45]. More 
importantly, this model exhibits relevant clinical TBI symptoms [46]. The present study demonstrated that $\mathrm{CCl}$ can cause brain edema, BBB disruption, an inflammatory response, and neurofunctional behavioral deficits after acute TBI, which can be assessed in the Garcia and rotarod tests.

The pathology of TBI is highly heterogeneous, and TBI manifests in a variety of ways that are caused by both immediate and delayed mechanisms. Immediate primary injury is related to impact and considered untreatable but preventable. In contrast, the reciprocal pathology of the delayed second phase of injury, in which rapid BBB disruption increases the levels of proinflammatory cytokines in the brain, causing further BBB function loss after exposure to these proinflammatory cytokines, allows a window of time for intervention [47]. Once initiated, diverse and intertwined neuroinflammatory processes are induced by and lead to BBB breakdown [47]. Several studies have reported that BBB dysfunction leads to a high degree of cerebral edema, increases the mortality rate and changes behavior [21, 48]. Thus, preventing inflammation of the BBB and protecting the BBB in the meantime might represent a prudent two-target therapeutic strategy. Based on the current study, we speculate that FGF20 can ameliorate neurofunctional behavioral deficits and decrease the degree of brain edema and neuronal apoptosis via protecting the BBB and reducing the inflammatory response after TBI.

Several studies have indicated that proinflammatory cytokines are related to BBB dysregulation and neurological disorders [49]. As a proinflammatory cytokine, TNF-a contributes to systemic inflammation, especially in the acute phase of BBB disruption [50]. Many studies have illustrated the ability of TNF-a to increase paracellular permeability in brain microvascular ECs and reduce TJ protein expression [51]. To study the protective effect of rhFGF20 against BBB damage, an in vitro TNF-a-induced BBB disruption model was used in our study. TNF-a-treated HBMECs exhibited increased paracellular permeability in the FITC-dextran extravasation and TEER experiments and the reduced expression of junction proteins; however, TNF-a did not change cell viability. Our results illustrated that TNF-a treatment successfully established an in vitro model of BBB disruption in HBMEC, which is consistent with a previous study [21, 27].

FGF20 and its family members are expressed in both embryonic and adult tissues, suggesting that they are critical factors in brain development and cell homeostasis [52]. Previous papers have shown that the expression levels of FGF20 may be vital to a signaling cascade involved in the repair of damaged dopaminergic neurons [17]. However, there is no information with respect to the effect of FGF20 following TBI. In the current study, we found that TBI was closely correlated with FGF20 and that intranasal FGF20 administration could deliver FGF20 to the brain. Further research through both in vitro and in vivo experiments illustrated that FGF20 prevented BBB disruption and decreased the inflammatory response to repair damaged ECs, thereby ameliorating the degree of edema and neurofunctional behavioral deficits.

FGF20 is also associated with 5-HT and depressive symptoms in adults [53]. Notably, some patients experience anhedonia and despair after TBI [54]. We speculated that FGF20 can ameliorate neurological deficits following TBI and that exogenous FGF20 can accelerate skull healing after TBI because of the 
important role of FGF20 in skull closure observed in developing mice[55]. According to previous research, exogenous FGF20 promoted the differentiation of human embryonic stem cells into neurons[55]. Therefore, it is worth noting the desirable prospects of FGF20 for treating TBI.

The AKT pathway is necessary for the mediation of cell survival and apoptosis. Previous studies have demonstrated that p-AKT protects against BBB damage after TBI. The phosphorylation of AKT was shown to decrease GSK3 activity [34] via an increase in the phosphorylation of GSK33. GSK3 $\beta$ deactivation resulted in the increased expression of the neuroprotective and neurotrophic proteins [35] brain-derived neurotrophic factor (BDNF) [56] and heat-shock protein 70 (HSP70) and attenuated BBB recovery [57]. Previous studies have illustrated that aFGF and bFGF protect against BBB injury through regulating junction proteins via the AKT-Rac1 pathway[26]. However, the definitive mechanism of rhFGF20 and the AKT pathway in the CNS has not been clarified. In the current study, both in vivo and in vitro models were used to illustrate that rhFGF20 significantly increased p-AKT and p-GSK3ß, consequently activating the AKT/GSK3 $\beta$ pathway. These findings are consistent with a previous study in which the protective effects of FGF20 were shown to be initiated in human umbilical vein endothelial cells (HUVECs) by AKT activation [58]. To further illustrate the effect of AKT/GSK3 $\beta$ pathway activation by rhFGF20, the AKT inhibitor LY294002 and GSK3 $\beta$ inhibitor SB216763 were used, and rhFGF20 was demonstrated to have a protective effect on BBB integrity through upregulating $\mathrm{TJ}$ and $\mathrm{AJ}$ protein expression by the AKT/GSK3 $\beta$ pathway.

As a central regulator of the inflammatory process, NFKB plays a pivotal role in inflammation and may be an optimal therapeutic target for the pathogenesis of inflammation [59]. NFKB regulates the expression of proinflammatory cytokines, chemokines and a whole host of adhesion molecules [60]. Microglia can remain chronically activated after TBI via the NFKB pathway [61], potentially contributing to neuroinflammatory and progressive neurodegeneration, making balancing their activation a potentially useful strategy over both the short and long term. JNK plays an important role in inflammation and can regulate the transcription of various inflammatory cytokines[61]. However, the interaction between JNK and NFKB remains unclear. In a previous study, JNK most likely acted upstream of NFKB in diabetic mice [62]. However, research is needed to determine whether JNK regulates the activity of NFKB in the CNS. In our study, rhFGF20 decreased the ratios of p-JNK/JNK and p-NFKB p65/NFKB p65 in mouse brains after TBI. Moreover, in vitro experiments showed that the JNK agonist anisomycin increased the expression of p-NFKB p65 and proinflammatory cytokines after rhFGF20 treatment, suggesting that changes in NFKB activity were a consequence of JNK. In addition, rhFGF20 inhibited JNK in early stage of TBI to inhibit the NFKB-mediated transcription of inflammatory cytokines, preventing further BBB disruption.

rhFGF20 was found to protect against BBB disruption through upregulating junction protein expression via the AKT/GSK3 $\beta$ pathway and decreasing the inflammatory response via the JNK/NFKB pathway. In our research, we found that the NFKB inhibitor Bay 11-7082 also protected against TNF-a-induced BBB injury in terms of changes to BBB permeability and endothelial resistance, which is consistent with a previous study showing that the NFKB pathway plays an important role in BBB disruption [63]. Notably, the protective effect of rhFGF20 was better than that of Bay 11-7082. This could be because the two- 
target therapeutic mechanism by which rhFGF20 protects against BBB was rapidly disrupted through increased TJ protein expression, thereby decreasing proinflammatory cytokine levels in the brain and preventing the inflammatory response, further protecting BBB function. This finding suggests that rhFGF20 may be an effective therapeutic target and represent an effective treatment strategy for TBI.

In conclusion, we first demonstrated that FGF20 is closely related to TBI. Exogenous rhFGF20 treatment facilitated neurofunctional recovery, relieved the degree of brain edema and protected against BBB disruption. Moreover, rhFGF20 increased TJ and AJ protein expression to protect BBB function via the AKT/GSK3 $\beta$ pathway. In addition, rhFGF20 inhibited the cerebral inflammatory response through regulating the JNK/NFKB pathway and further protected the function of the BBB (Fig. 11). Taken together, our results suggest that the two-target therapeutic mechanism of rhFGF20 may exert efficient neuroprotective effects following TBI.

\section{Abbreviations}

FGF20:fibroblast growth factor 20; BBB:blood-brain barrier; TBI:traumatic brain injury; PD:Parkinson disease; CCl:controlled cortical impact; HBMEC:human brain microvascular endothelial cell; rhFGF20:recombinant human fibroblast growth factor20; TEER:transendothelial electrical resistance; TJ:tight junction; AJ:adherence junction; AKT:protein kinase B; GSK3ß:glycogen synthase kinase 3 beta; JNK:C-Jun N-terminal kinase; NFKB $\square$ nuclear factor kappa-B; EC:endothelial cell; ZO-1:zonula occludens-1; CNS:central nervous system; TNF-a:tumor necrosis factor-a; IL-1ß:interleukin-1 $\beta$; IL-6:interleukin-6; iNOS:inducible nitric oxide synthase; SNpc:substantia nigra pars compacta; H\&E:hematoxylin and eosin

\section{Declarations}

\section{Authors' contributions}

$\mathrm{LL}$ and $\mathrm{XKL}$ designed the experiments, $\mathrm{JC}$ and $\mathrm{XW}$ were involved in the design of the described experiments. JC, JH, WTH, CD, KMG, FYH and RLG carried out the experiments. SSY and JTD analyzed data, compiled the figures. JC wrote the manuscript, XW and LL reviewed the manuscript. All authors read and approved the final manuscript.

\section{Acknowledgements}

This work was supported by the by the National Natural Science Foundation of China (No. 81971180), Medical and Health Science and Technology Program of Zhejiang Province (2018267872), and the Wenzhou Municipal Science and Technology Bureau (Y20170686).

\section{Funding}

National Natural Science Foundation of China (No. 81971180), Medical and Health Science and Technology Program of Zhejiang Province (2018267872), and the Wenzhou Municipal Science and 
Technology Bureau (Y20170686).

Availability of data and materials

The datasets used and/or analyzed during the current study are available from the corresponding author on reasonable request.

Consent for publication

N/A

Competing interests

The authors declare that they have no competing interests

Ethics approval and consent to participate

The experimental protocols in the present study including all surgical procedures and animal usages conformed to the guidelines for the care and use of laboratory animals by the National Institutes of Health $(\mathrm{NIH})$ and were approved by the Wenzhou Medical University Experimental Animal Ethics Committee (Wenzhou, China).

\section{References}

1. Maas Al, Stocchetti N, Bullock R: Moderate and severe traumatic brain injury in adults. Lancet Neurol 2008, 7:728-741.

2. Runyan DK: The challenges of assessing the incidence of inflicted traumatic brain injury: a world perspective. Am J Prev Med 2008, 34:S112-115.

3. Loane DJ, Faden Al: Neuroprotection for traumatic brain injury: translational challenges and emerging therapeutic strategies. Trends Pharmacol Sci 2010, 31:596-604.

4. Polderman KH: Application of therapeutic hypothermia in the ICU: opportunities and pitfalls of a promising treatment modality. Part 1: Indications and evidence. Intensive Care Med 2004, 30:556575.

5. Bramlett HM, Dietrich WD: Progressive damage after brain and spinal cord injury: pathomechanisms and treatment strategies. Prog Brain Res 2007, 161:125-141.

6. Shi H, Wang HL, Pu HJ, Shi YJ, Zhang J, Zhang WT, Wang GH, Hu XM, Leak RK, Chen J, Gao YQ:

Ethyl pyruvate protects against blood-brain barrier damage and improves long-term neurological outcomes in a rat model of traumatic brain injury. CNS Neurosci Ther 2015, 21:374-384.

7. Abbott NJ, Ronnback L, Hansson E: Astrocyte-endothelial interactions at the blood-brain barrier. Nat Rev Neurosci 2006, 7:41-53.

8. Zhao Z, Nelson AR, Betsholtz C, Zlokovic BV: Establishment and Dysfunction of the Blood-Brain Barrier. Cell 2015, 163:1064-1078. 
9. Butt AM, Jones HC, Abbott NJ: Electrical resistance across the blood-brain barrier in anaesthetized rats: a developmental study. J Physio/ 1990, 429:47-62.

10. Jiao H, Wang Z, Liu Y, Wang P, Xue Y: Specific role of tight junction proteins claudin-5, occludin, and Z0-1 of the blood-brain barrier in a focal cerebral ischemic insult. J Mol Neurosci 2011, 44:130-139.

11. Simon DW, McGeachy MJ, Bayir H, Clark RS, Loane DJ, Kochanek PM: The far-reaching scope of neuroinflammation after traumatic brain injury. Nat Rev Neurol 2017, 13:171-191.

12. Corps KN, Roth TL, McGavern DB: Inflammation and neuroprotection in traumatic brain injury. JAMA Neurol 2015, 72:355-362.

13. Villalba N, Sonkusare SK, Longden TA, Tran TL, Sackheim AM, Nelson MT, Wellman GC, Freeman K: Traumatic brain injury disrupts cerebrovascular tone through endothelial inducible nitric oxide synthase expression and nitric oxide gain of function. J Am Heart Assoc 2014, 3:e001474.

14. Kamm K, Vanderkolk W, Lawrence C, Jonker M, Davis AT: The effect of traumatic brain injury upon the concentration and expression of interleukin-1beta and interleukin-10 in the rat. J Trauma 2006, 60:152-157.

15. Aibiki M, Maekawa S, Ogura S, Kinoshita Y, Kawai N, Yokono S: Effect of moderate hypothermia on systemic and internal jugular plasma IL-6 levels after traumatic brain injury in humans. J Neurotrauma 1999, 16:225-232.

16. Elwood E, Lim Z, Naveed H, Galea I: The effect of systemic inflammation on human brain barrier function. Brain Behav Immun 2017, 62:35-40.

17. Boshoff EL, Fletcher EJR, Duty S: Fibroblast growth factor 20 is protective towards dopaminergic neurons in vivo in a paracrine manner. Neuropharmacology 2018, 137:156-163.

18. Shimada H, Yoshimura N, Tsuji A, Kunisada T: Differentiation of dopaminergic neurons from human embryonic stem cells: modulation of differentiation by FGF-20. J Biosci Bioeng 2009, 107:447-454.

19. Takagi Y, Takahashi J, Saiki H, Morizane A, Hayashi T, Kishi Y, Fukuda H, Okamoto Y, Koyanagi M, Ideguchi $\mathrm{M}$, et al: Dopaminergic neurons generated from monkey embryonic stem cells function in a Parkinson primate model. J Clin Invest 2005, 115:102-109.

20. Niu J, Xie J, Guo K, Zhang X, Xia F, Zhao X, Song L, Zhuge D, Li X, Zhao Y, Huang Z: Efficient treatment of Parkinson's disease using ultrasonography-guided rhFGF20 proteoliposomes. Drug Deliv 2018, 25:1560-1569.

21. Chen J, Hu J, Liu H, Xiong Y, Zou Y, Huang W, Shao M, Wu J, Yu L, Wang X, et al: FGF21 Protects the Blood-Brain Barrier by Upregulating PPARgamma via FGFR1/beta-klotho after Traumatic Brain Injury. J Neurotrauma 2018, 35:2091-2103.

22. Sabir M, Gaudreault PO, Freyburger M, Massart R, Blanchet-Cohen A, Jaber M, Gosselin N, Mongrain $\mathrm{V}$ : Impact of traumatic brain injury on sleep structure, electrocorticographic activity and transcriptome in mice. Brain Behav Immun 2015, 47:118-130.

23. Chen X, Wu S, Chen C, Xie B, Fang Z, Hu W, Chen J, Fu H, He H: Omega-3 polyunsaturated fatty acid supplementation attenuates microglial-induced inflammation by inhibiting the HMGB1/TLR4/NFkappaB pathway following experimental traumatic brain injury. J Neuroinflammation 2017, 14:143. 
24. Garcia JH, Wagner S, Liu KF, Hu XJ: Neurological deficit and extent of neuronal necrosis attributable to middle cerebral artery occlusion in rats. Statistical validation. Stroke 1995, 26:627-634; discussion 635.

25. Glushakov AV, Robbins SW, Bracy CL, Narumiya S, Dore S: Prostaglandin F2alpha FP receptor antagonist improves outcomes after experimental traumatic brain injury. J Neuroinflammation 2013, 10:132.

26. Wang ZG, Cheng Y, Yu XC, Ye LB, Xia QH, Johnson NR, Wei X, Chen DQ, Cao G, Fu XB, et al: bFGF Protects Against Blood-Brain Barrier Damage Through Junction Protein Regulation via PI3K-Akt-Rac1 Pathway Following Traumatic Brain Injury. Mol Neurobio/ 2016, 53:7298-7311.

27. Rochfort KD, Cummins PM: Cytokine-mediated dysregulation of zonula occludens-1 properties in human brain microvascular endothelium. Microvasc Res 2015, 100:48-53.

28. Li L, Shao X, Cole EL, Ohnmacht SA, Ferrari V, Hong YT, Williamson DJ, Fryer TD, Quesada CA, Sherman $P$, et al: Synthesis and Initial in Vivo Studies with [(11)C]SB-216763: The First Radiolabeled Brain Penetrative Inhibitor of GSK-3. ACS Med Chem Lett 2015, 6:548-552.

29. Li JY, Huang JY, Li M, Zhang H, Xing B, Chen G, Wei D, Gu PY, Hu WX: Anisomycin induces glioma cell death via down-regulation of PP2A catalytic subunit in vitro. Acta Pharmacol Sin 2012, 33:935-940.

30. Odobasic D, Oudin V, Ito K, Gan PY, Kitching AR, Holdsworth SR: Tolerogenic Dendritic Cells Attenuate Experimental Autoimmune Antimyeloperoxidase Glomerulonephritis. J Am Soc Nephrol 2019.

31. Zhao Q, Zhang F, Yu Z, Guo S, Liu N, Jiang Y, Lo EH, Xu Y, Wang X: HDAC3 inhibition prevents bloodbrain barrier permeability through Nrf2 activation in type 2 diabetes male mice. J Neuroinflammation 2019, 16:103.

32. Das M, Tang X, Han JY, Mayilsamy K, Foran E, Biswal MR, Tzekov R, Mohapatra SS, Mohapatra S: CCL20-CCR6 axis modulated traumatic brain injury-induced visual pathologies. J Neuroinflammation 2019, 16:115.

33. Noshita N, Lewen A, Sugawara T, Chan PH: Akt phosphorylation and neuronal survival after traumatic brain injury in mice. Neurobiol Dis 2002, 9:294-304.

34. Zhao H, Sapolsky RM, Steinberg GK: Phosphoinositide-3-kinase/akt survival signal pathways are implicated in neuronal survival after stroke. Mol Neurobio/ 2006, 34:249-270.

35. Ren M, Senatorov VV, Chen RW, Chuang DM: Postinsult treatment with lithium reduces brain damage and facilitates neurological recovery in a rat ischemia/reperfusion model. Proc Natl Acad Sci U S A 2003, 100:6210-6215.

36. Ramirez SH, Fan S, Dykstra H, Rom S, Mercer A, Reichenbach NL, Gofman L, Persidsky Y: Inhibition of glycogen synthase kinase 3beta promotes tight junction stability in brain endothelial cells by halflife extension of occludin and claudin-5. PLoS One 2013, 8:e55972.

37. Zhou Y, Ming J, Li Y, Deng M, Chen Q, Ma Y, Chen Z, Zhang Y, Liu S: Ligustilide attenuates nitric oxideinduced apoptosis in rat chondrocytes and cartilage degradation via inhibiting JNK and p38 MAPK pathways. J Cell Mol Med 2019, 23:3357-3368. 
38. Oikawa S, Kai Y, Mano A, Sugama S, Mizoguchi N, Tsuda M, Muramoto K, Kakinuma Y: Potentiating a non-neuronal cardiac cholinergic system reinforces the functional integrity of the blood brain barrier associated with systemic anti-inflammatory responses. Brain Behav Immun 2019.

39. Lu S, Luo Y, Zhou P, Yang K, Sun G, Sun X: Ginsenoside compound K protects human umbilical vein endothelial cells against oxidized low-density lipoprotein-induced injury via inhibition of nuclear factor-kappaB, p38, and JNK MAPK pathways. J Ginseng Res 2019, 43:95-104.

40. Beauchamp K, Mutlak H, Smith WR, Shohami E, Stahel PF: Pharmacology of traumatic brain injury: where is the "golden bullet"? Mol Med 2008, 14:731-740.

41. Maas Al, Roozenbeek B, Manley GT: Clinical trials in traumatic brain injury: past experience and current developments. Neurotherapeutics 2010, 7:115-126.

42. Bales JW, Wagner AK, Kline AE, Dixon CE: Persistent cognitive dysfunction after traumatic brain injury: A dopamine hypothesis. Neurosci Biobehav Rev 2009, 33:981-1003.

43. Shelton SB, Pettigrew DB, Hermann AD, Zhou W, Sullivan PM, Crutcher KA, Strauss KI: A simple, efficient tool for assessment of mice after unilateral cortex injury. J Neurosci Methods 2008, 168:431-442.

44. Prabhakara KS, Kota DJ, Jones GH, Srivastava AK, Cox CS, Jr., Olson SD: Teriflunomide Modulates Vascular Permeability and Microglial Activation after Experimental Traumatic Brain Injury. Mol Ther 2018, 26:2152-2162.

45. Peterson TC, Maass WR, Anderson JR, Anderson GD, Hoane MR: A behavioral and histological comparison of fluid percussion injury and controlled cortical impact injury to the rat sensorimotor cortex. Behav Brain Res 2015, 294:254-263.

46. Cernak I: Animal models of head trauma. NeuroRx 2005, 2:410-422.

47. Shlosberg D, Benifla M, Kaufer D, Friedman A: Blood-brain barrier breakdown as a therapeutic target in traumatic brain injury. Nat Rev Neuro/ 2010, 6:393-403.

48. Xu ZM, Yuan F, Liu YL, Ding J, Tian HL: Glibenclamide Attenuates Blood-Brain Barrier Disruption in Adult Mice after Traumatic Brain Injury. J Neurotrauma 2017, 34:925-933.

49. Tuttolomondo A, Di Raimondo D, Pecoraro R, Arnao V, Pinto A, Licata G: Inflammation in ischemic stroke subtypes. Curr Pharm Des 2012, 18:4289-4310.

50. Shohami E, Ginis I, Hallenbeck JM: Dual role of tumor necrosis factor alpha in brain injury. Cytokine Growth Factor Rev 1999, 10:119-130.

51. Schubert-Unkmeir A, Konrad C, Slanina H, Czapek F, Hebling S, Frosch M: Neisseria meningitidis induces brain microvascular endothelial cell detachment from the matrix and cleavage of occludin: a role for MMP-8. PLoS Pathog 2010, 6:e1000874.

52. Dono R: Fibroblast growth factors as regulators of central nervous system development and function. Am J Physiol Regul Integr Comp Physiol 2003, 284:R867-881.

53. Jimenez KM, Pereira-Morales AJ, Adan A, Lopez-Leon S, Forero DA: Depressive symptoms are associated with a functional polymorphism in a miR-433 binding site in the FGF20 gene. Mol Brain 
$2018,11: 53$.

54. Shultz SR, McDonald SJ, Corrigan F, Semple BD, Salberg S, Zamani A, Jones NC, Mychasiuk R: The Clinical Relevance of Behavior Testing in Animal Models of Traumatic Brain Injury. J Neurotrauma 2019.

55. Hajihosseini MK, Heath JK: Expression patterns of fibroblast growth factors-18 and -20 in mouse embryos is suggestive of novel roles in calvarial and limb development. Mech Dev 2002, 113:79-83.

56. Yasuda S, Liang MH, Marinova Z, Yahyavi A, Chuang DM: The mood stabilizers lithium and valproate selectively activate the promoter IV of brain-derived neurotrophic factor in neurons. Mol Psychiatry $2009,14: 51-59$

57. Yu F, Wang Z, Tchantchou F, Chiu CT, Zhang Y, Chuang DM: Lithium ameliorates neurodegeneration, suppresses neuroinflammation, and improves behavioral performance in a mouse model of traumatic brain injury. J Neurotrauma 2012, 29:362-374.

58. Maclachlan T, Narayanan B, Gerlach VL, Smithson G, Gerwien RW, Folkerts O, Fey EG, Watkins B, Seed T, Alvarez E: Human fibroblast growth factor 20 (FGF-20; CG53135-05): a novel cytoprotectant with radioprotective potential. Int J Radiat Biol 2005, 81:567-579.

59. Lan YL, Wang X, Zou YJ, Xing JS, Lou JC, Zou S, Ma BB, Ding Y, Zhang B: Bazedoxifene protects cerebral autoregulation after traumatic brain injury and attenuates impairments in blood-brain barrier damage: involvement of anti-inflammatory pathways by blocking MAPK signaling. Inflamm Res 2019, 68:311-323.

60. Rahman A, Fazal F: Blocking NF-kappaB: an inflammatory issue. Proc Am Thorac Soc 2011, 8:497503.

61. Johnson VE, Stewart JE, Begbie FD, Trojanowski JQ, Smith DH, Stewart W: Inflammation and white matter degeneration persist for years after a single traumatic brain injury. Brain 2013, 136:28-42.

62. Liang G, Song L, Chen Z, Qian Y, Xie J, Zhao L, Lin Q, Zhu G, Tan Y, Li X, et al: Fibroblast growth factor 1 ameliorates diabetic nephropathy by an anti-inflammatory mechanism. Kidney Int 2018, 93:95-109.

63. McMahon D, Hynynen K: Acute Inflammatory Response Following Increased Blood-Brain Barrier Permeability Induced by Focused Ultrasound is Dependent on Microbubble Dose. Theranostics 2017, 7:3989-4000.

\section{Figures}




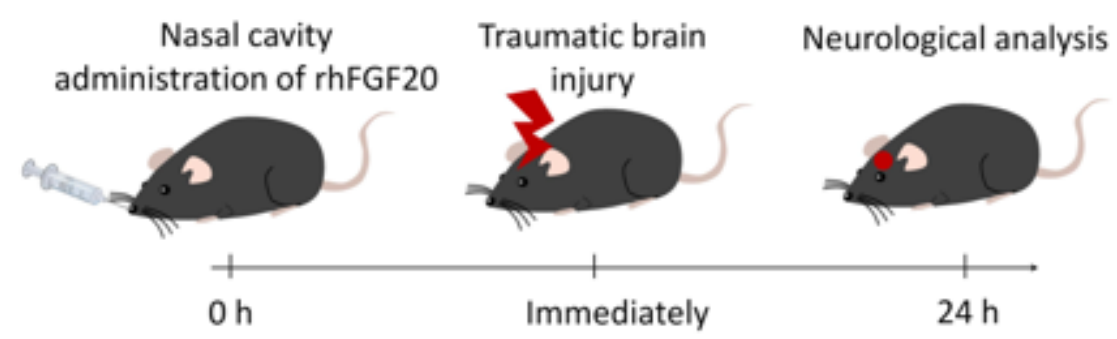

B

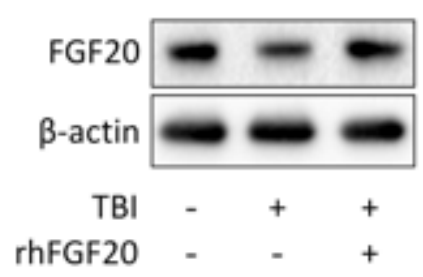

C

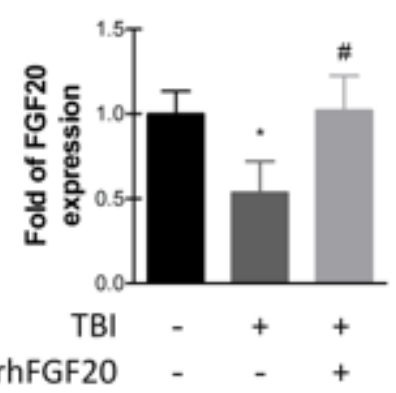

D

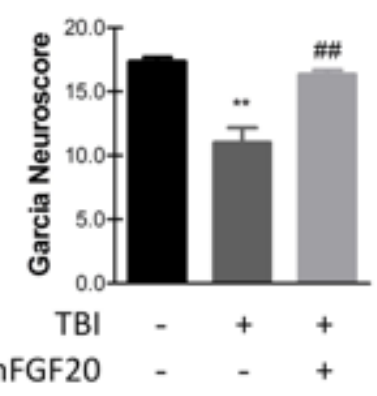

E

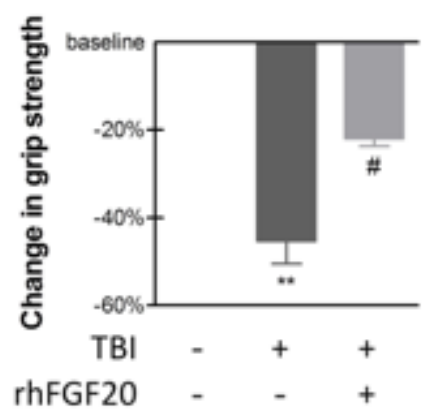

$\mathbf{F}$

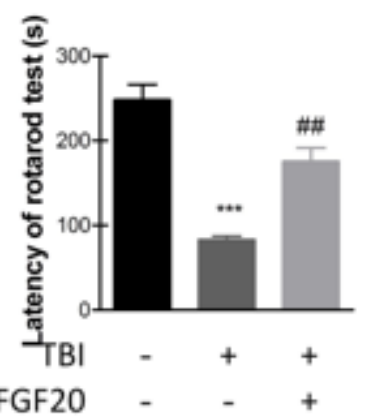

G

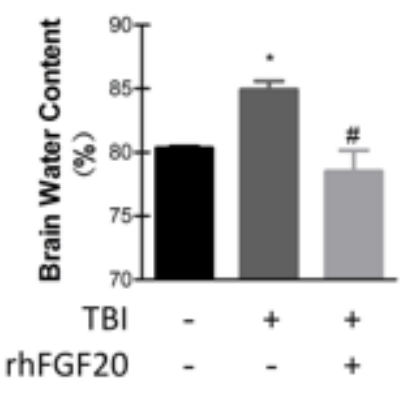

\section{Figure 1}

Exogenous rhFGF20 improves neurofunctional deficits and ameliorates TBI parameters in mice. (A) A schematic diagram showing the in vivo experimental protocol. (B) Representative image from western blot analysis of FGF20 in the sham, TBI, and rhFGF20 treatment groups. (C) Quantification of western blotting data. (D) Effects of rhFGF20 assessed by the Garcia test $24 \mathrm{~h}$ after TBI. (E) Effects of rhFGF20 assessed by the grip strength test $24 \mathrm{~h}$ after TBI. (F) Effects of rhFGF20 assessed by the rotarod test $24 \mathrm{~h}$ after TBI. (G) Effects of rhFGF20 on brain water content $24 \mathrm{~h}$ after TBI. ${ }^{*} \mathrm{P}<0.05,{ }^{\star} * \mathrm{P}<0.01,{ }^{\star} * \star \mathrm{P}<0.001$ vs. sham group. \#P<0.05, \#\#P<0.01 vs. TBI group. Data are presented as the mean values $\pm S E M(n=$ 5). 


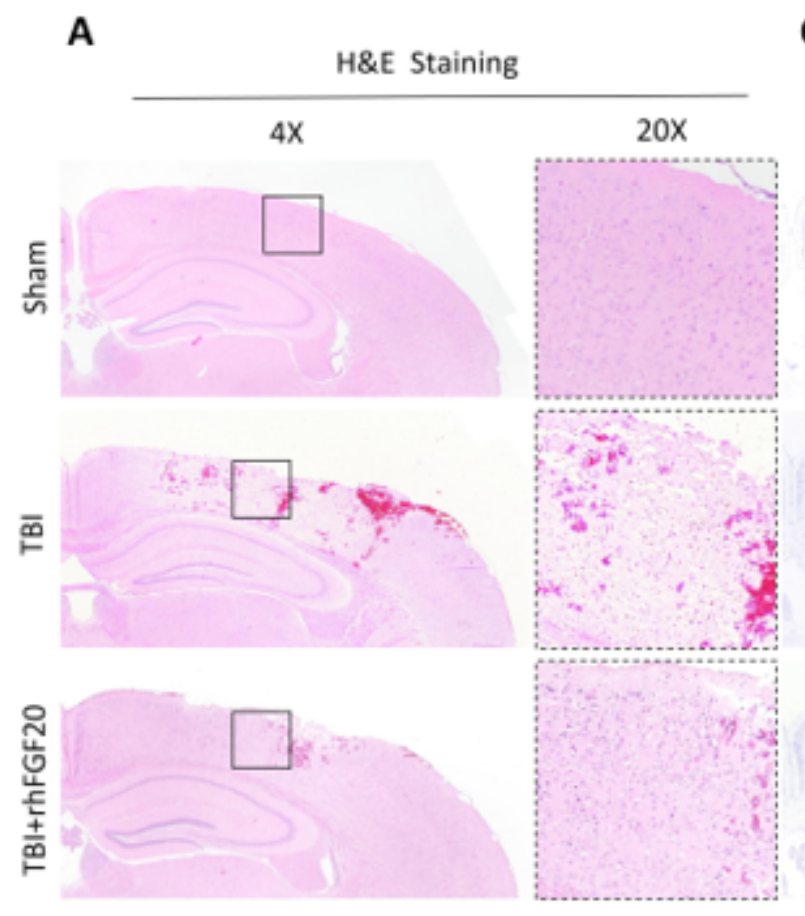

B

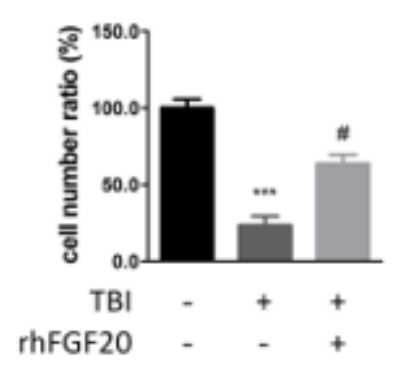

C

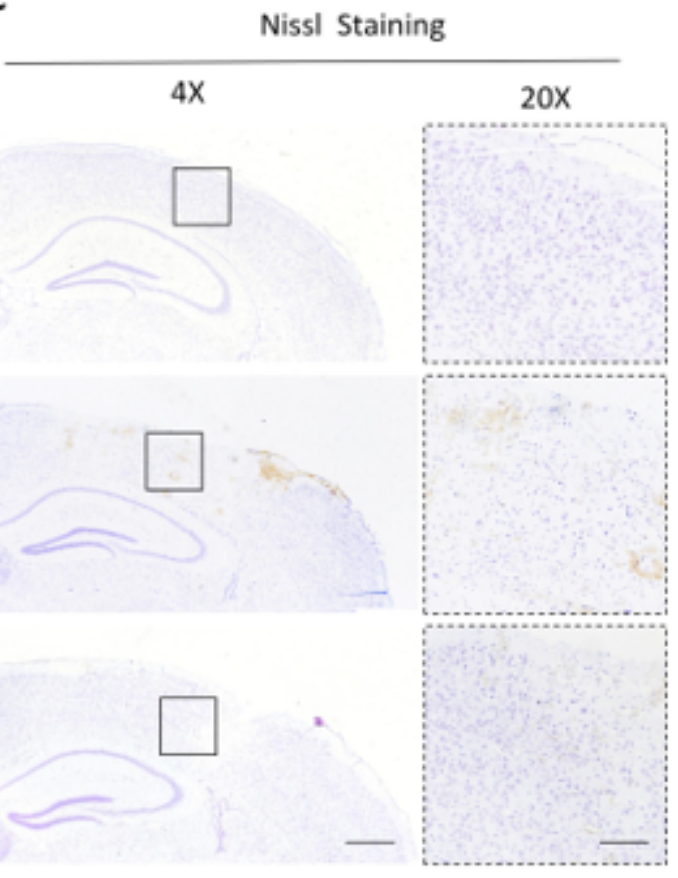

D

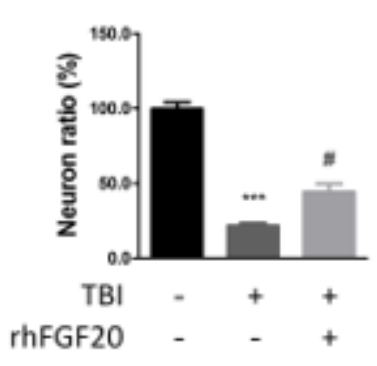

Figure 2

Exogenous rhFGF20 recues tissue degeneration in mouse brain at $24 \mathrm{~h}$ after TBI. (A) Effects of rhFGF20 on damaged brain tissue after TBI visualized with H\&E staining. (B) Quantification of H\&E staining data for cell number ratio. (C) Effects of rhFGF20 on neuron apoptosis in the brain after TBI demonstrated with Nissl staining. (D) Quantification of Nissl staining data for neuron number ratio. 4x: Bar, $500 \mu \mathrm{m}$. 20x: $B a r, 100 \mu m .{ }^{* \star *} P<0.001$ vs. sham group. $\# P<0.05$ vs. TBI group. Data are presented as the mean values $\pm \operatorname{SEM}(n=5)$. 
A

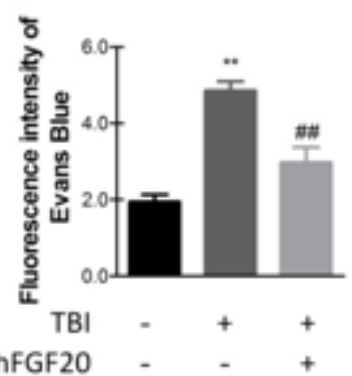

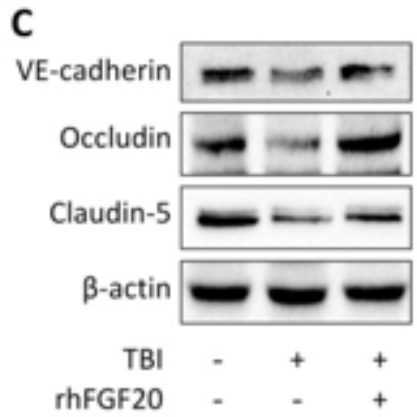

D

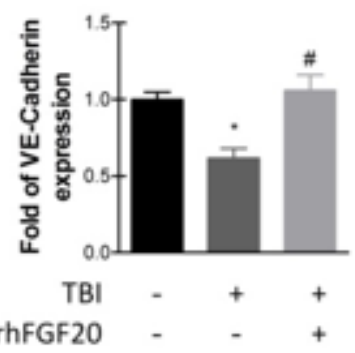

$\mathbf{E}$

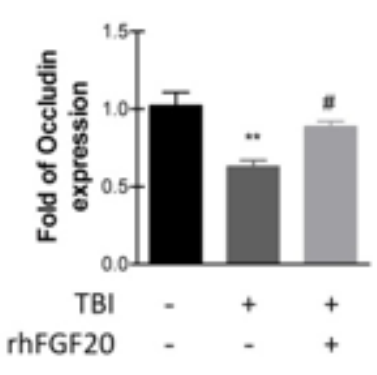

$\mathbf{F}$

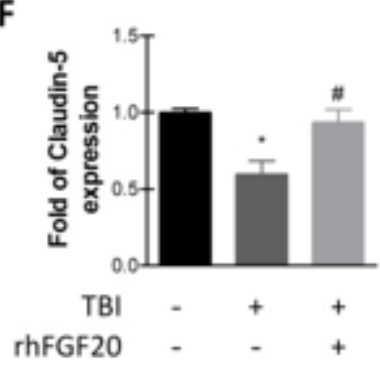

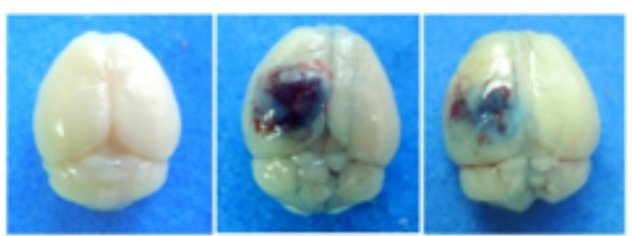

$\mathrm{TB} \mid$ rhFGF20

G
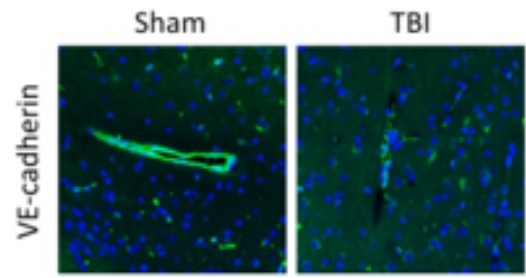

TBI+rhFGF20
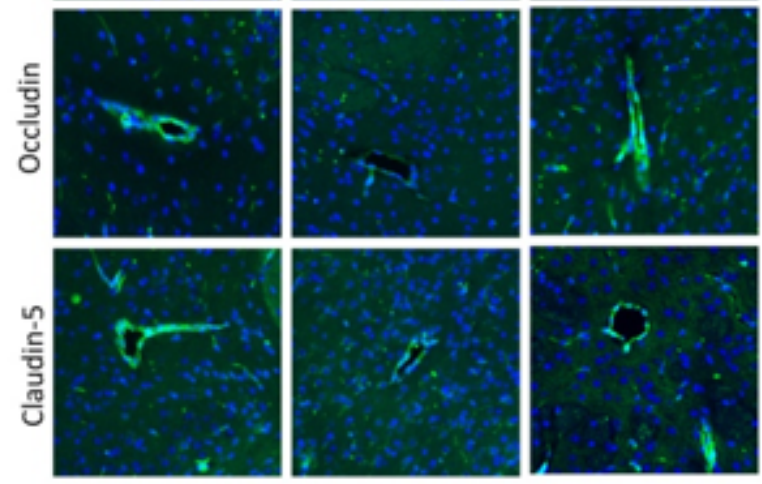

\section{Figure 3}

Exogenous rhFGF20 protects against BBB disruption in mouse brain at $24 \mathrm{~h}$ after TBI. (A) Effects of rhFGF20 on Evans blue fluorescence. (B) Representative effects of Evans blue extravasation. (C) Representative image of western blotting analysis of VE-Cadherin, Occludin, Claudin-5. $\beta$-Actin was used as the loading control and for band density normalization. (D-F) Quantification of western blotting data for VE-Cadherin, Occludin, and Claudin-5 protein. (G) Effects of rhFGF20 on the expression of the VECadherin, Occludin, and Claudin-5. The nuclei are labeled with DAPI. $n=5$. Bar, $50 \mu \mathrm{m}$. Magnification, $\times 40$. ${ }^{\star} \mathrm{P}<0.05,{ }^{*} \mathrm{P}<0.01$ vs. sham group. $\# \mathrm{P}<0.05, \# \# \mathrm{P}<0.01$ vs. TBI group. Data are presented as the mean values \pm SEM $(n=5)$. 
A

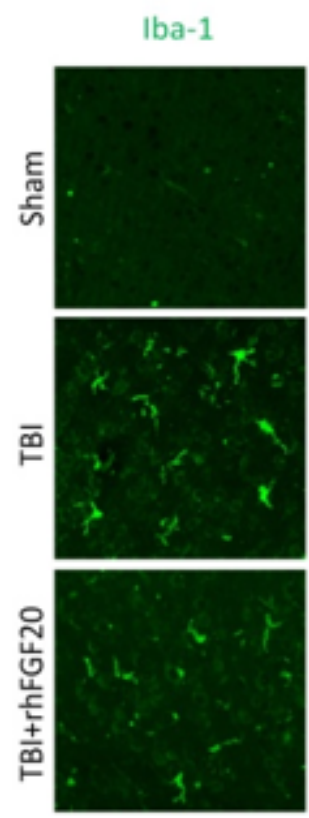

B

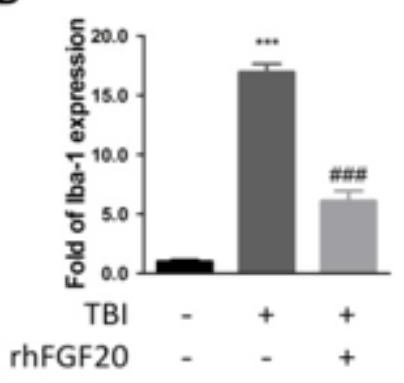

Dapi
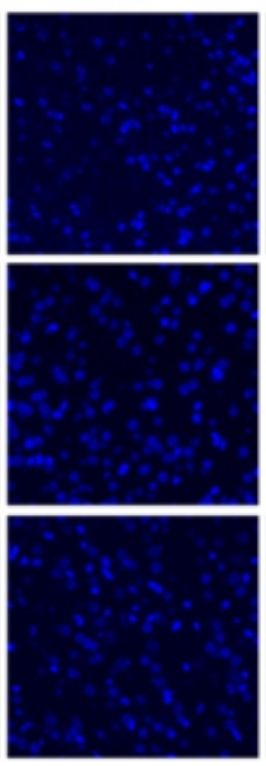

C

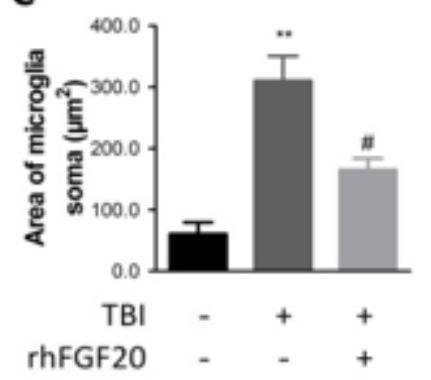

E

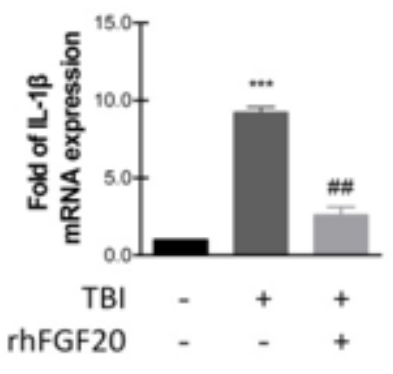

F

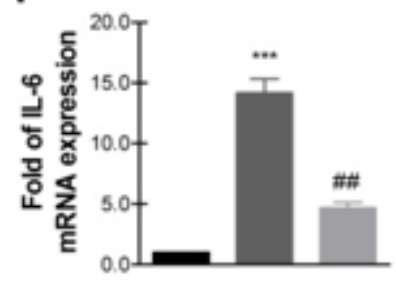

TBI

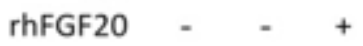

G

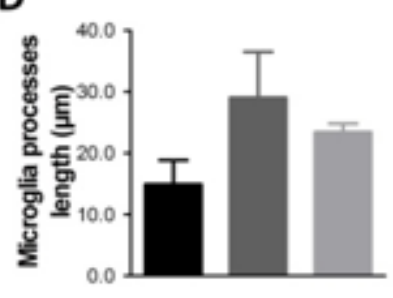

TBI hFGF20

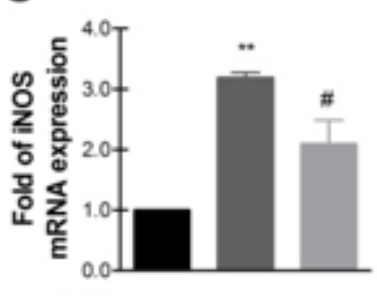

TBI

rhFGF2O

\section{Figure 4}

Exogenous rhFGF20 inhibits neuroinflammation and decreases the inflammatory response in the mouse brain at $24 \mathrm{~h}$ after TBI. (A) Effects of rhFGF20 on the activity of the lba-1 protein. Nuclei are labeled with DAPI. (B-D) Quantification of immunofluorescence staining data showing Iba-1 expression, microglia soma area and microglia process length. (E-G) Effects of rhFGF20 on mRNA levels of the proinflammatory cytokines IL-1 $\beta$, IL-6, and iNOS, with values normalized to the housekeeping gene $\beta$ -

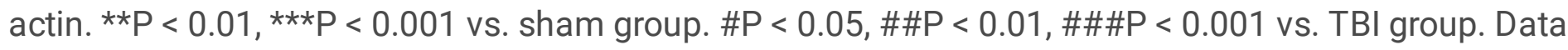
are presented as the mean values $\pm \operatorname{SEM}(n=5)$. 


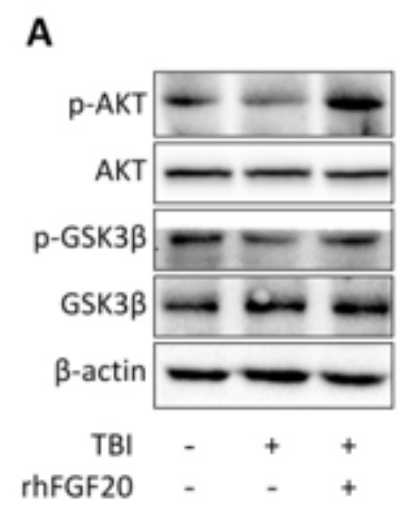

B
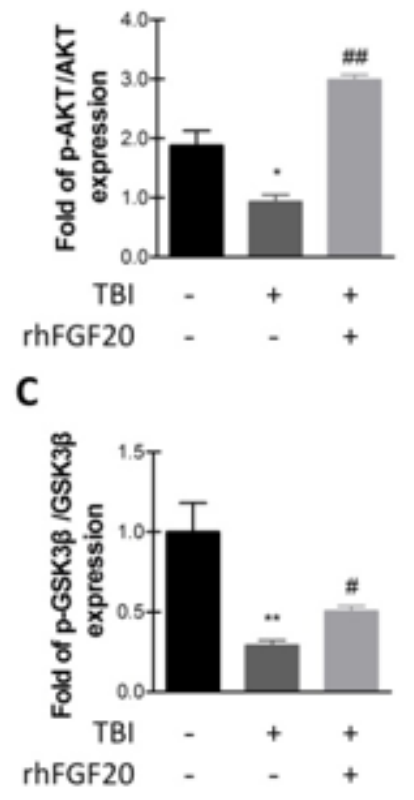

$\mathbf{E}$
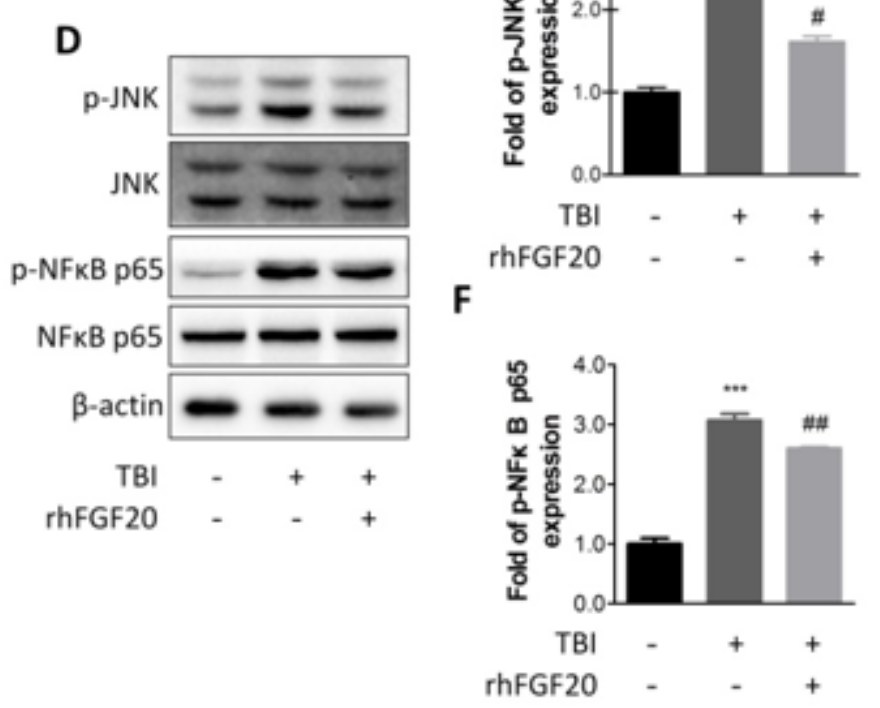

\section{Figure 5}

Exogenous rhFGF20 protects against TBl-induced BBB disruption via the AKT/GSK3 $\beta$ and JNK/NFKB pathways. (A) Representative image from western blot analysis of p-AKT, AKT, p-GSK3 $\beta$ and GSK3 $\beta$ in mouse brain at $24 \mathrm{~h}$ after TBI. (B-C) Effects of rhFGF20rhFGF20 on p-AKT, AKT, GSK3 $\beta$ and p-GSK3 $\beta$ protein analyzed by quantified western blotting data. (D) Representative image from western blot analysis of p-JNK, JNK, p-NFKB p65 and NFKB p65 in the sham, TBI and rhFGF20 treatment groups. $\beta$ Actin was used as the loading control and for band density normalization. (E-F) Effects of rhFGF20 on the p-JNK, JNK, p-NFKB p65 and NFKB p65 proteins analyzed by quantified western blotting data. ${ }^{*} \mathrm{P}<$ 0.05 , ${ }^{\star \star} P<0.01,{ }^{\star \star \star} \mathrm{P}<0.001$ vs. sham group. \#P $<0.05$, \#\#P $<0.01$ vs. TBI group. Data are presented as the mean values \pm SEM $(n=5)$. 
A

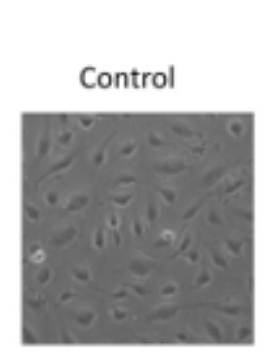

C

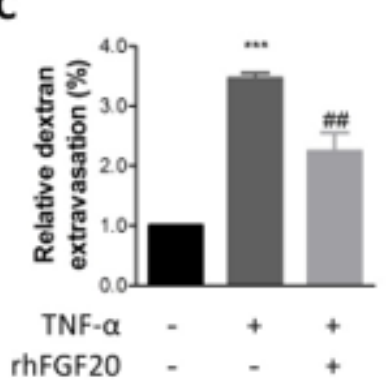

E

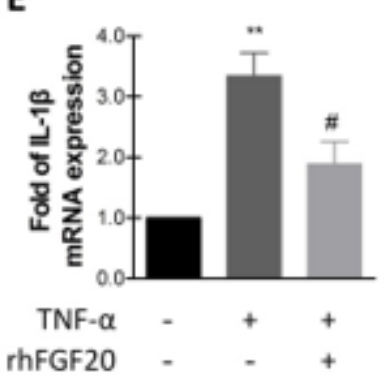

TNF- $\alpha$

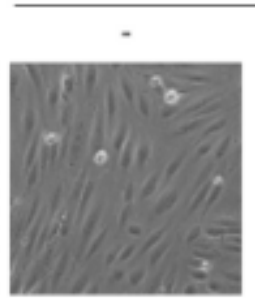

rhFGF20

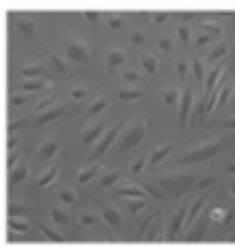

$\mathbf{F}$
B

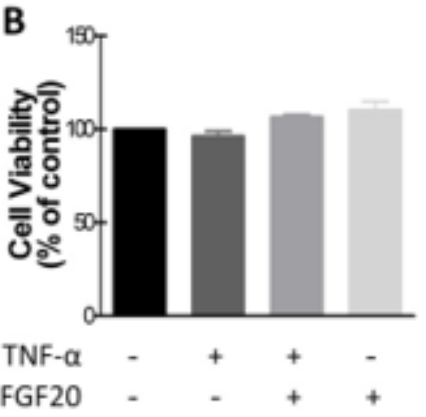

D

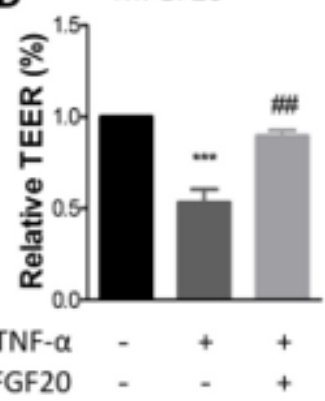

G
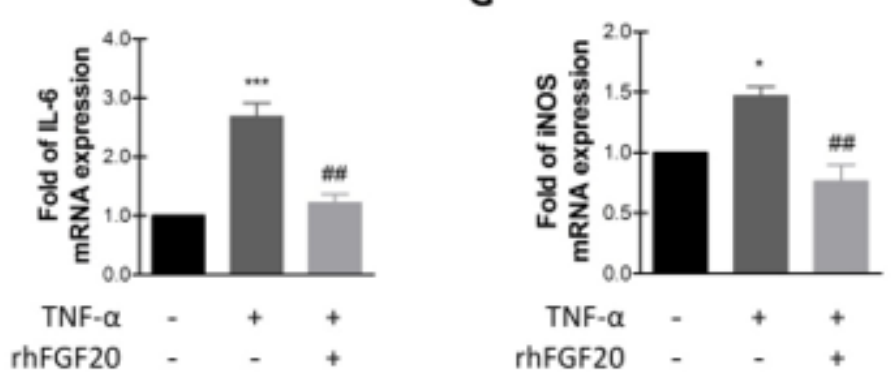

\section{Figure 6}

rhFGF20 rescues monolayers damage and decreases inflammatory response in TNF-a induced HBMEC. (A) Effects of TNF-a and rhFGF20 on HBMEC morphology. (B) Effects of TNF-a and rhFGF20 on HBMEC viability. (C) Effects of rhFGF20 on HBMECs FITC-dextran extravasation test. (D) Effects of rhFGF20 on TEER measurements. (E-G) Effects of rhFGF20 on mRNA of pro-inflammatory cytokines,IL-1 $\beta$, IL-6, and iNOS, values normalized to the housekeeping gene $\beta$-actin. ${ }^{*} P<0.05$, ${ }^{\star *} P<0.01$, $* \star * P<0.001$ vs. control group. \#P<0.05, \#\#P<0.01 vs. TNF-a group. Data are presented as the mean values \pm SEM $(n=5)$. 
A

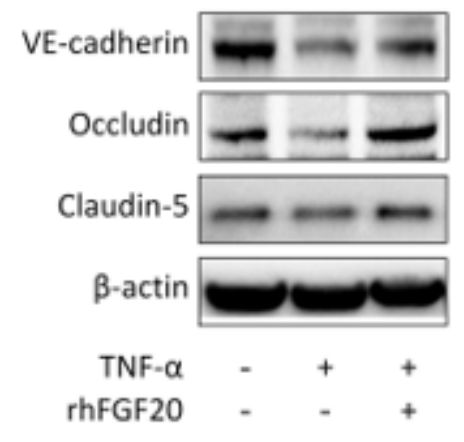

B

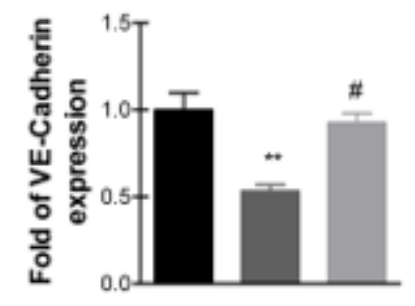

TNF- $\alpha$ - + +

rhFGF20 - - +
C

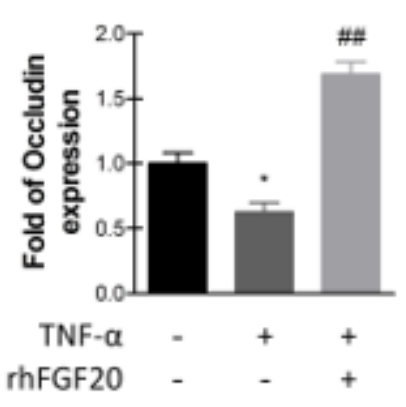

D

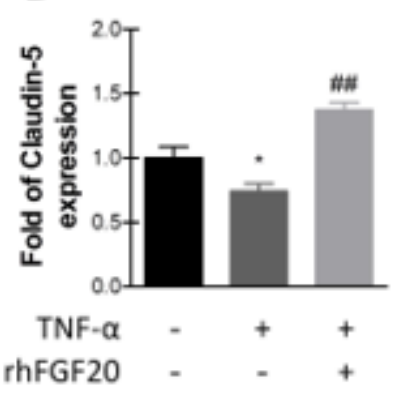

E

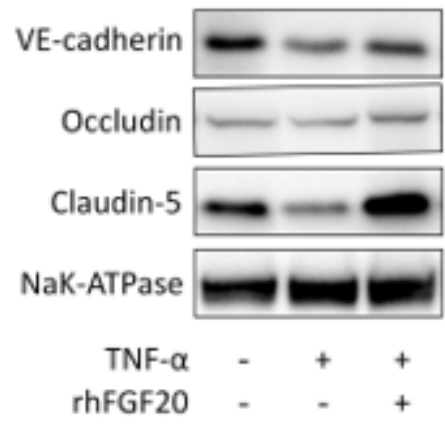

$\mathbf{F}$

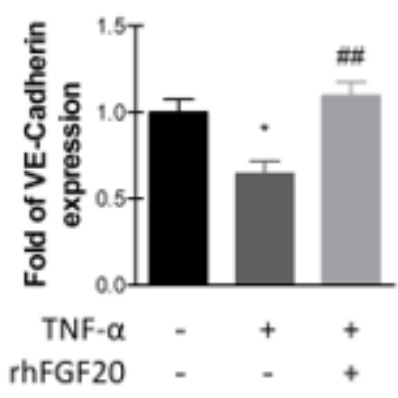

G

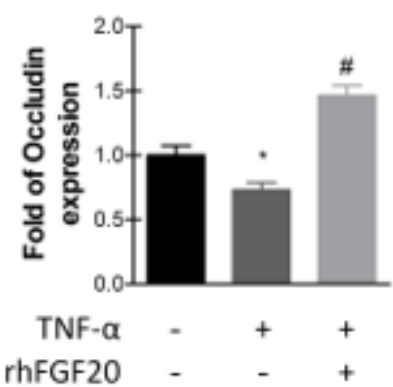

H

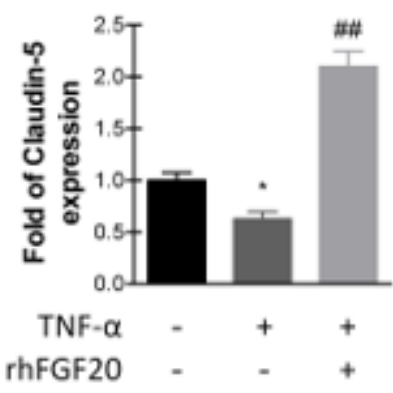

\section{Figure 7}

rhFGF20 rescues both total and membrane TJ protein expression in TNF-a induced HBMECS. (A) Effects of rhFGF20 on the expression of VE-Cadherin, Occludin, and Claudin- 5 by western blot analysis in total cellular protein. $\beta$-Actin was used as the loading control and for band density normalization. (B-D) Effects of rhFGF20 on VE-Cadherin, Occludin, and Claudin-5 protein levels analyzed by quantification of western blotting data of total protein. (E) Effects of rhFGF20 on the expression of VE-cadherin, Occludin, and Claudin- 5 by western blot analysis in cell membrane protein. NaK-ATPase was used as the loading control and for band density normalization. (F-H) Effects of rhFGF20 on VE-cadherin, Occludin, and Claudin- 5 protein levels analyzed by quantification of western blotting data of membrane proteins. ${ }^{*} \mathrm{P}<$ $0.05,{ }^{\star} \mathrm{P}<0.01$ vs. control group. $\# \mathrm{P}<0.05, \# \# \mathrm{P}<0.01$ vs. TNF-a group. Data are presented as the mean values $\pm \operatorname{SEM}(n=5)$. 
A

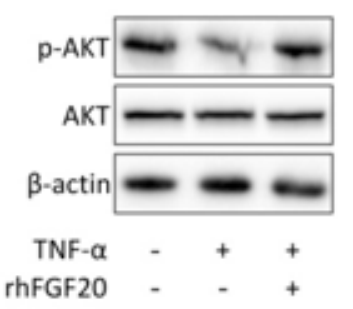

C

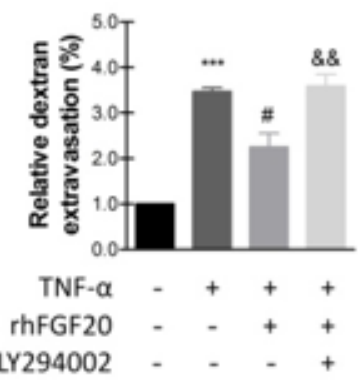

E

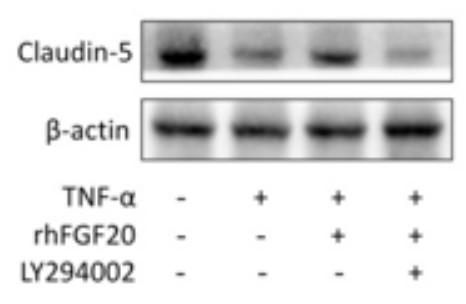

B

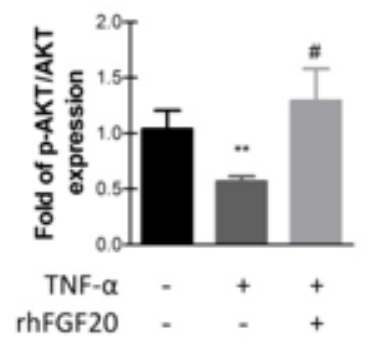

D

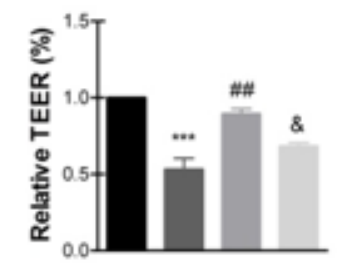

TNF- $\alpha-+++$ ITFGF20 - - + +

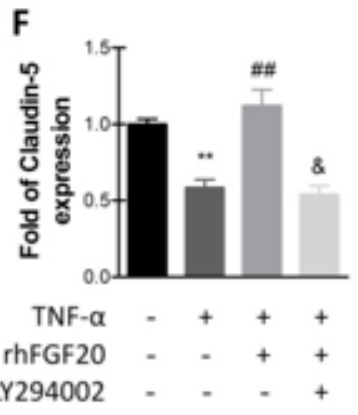

G
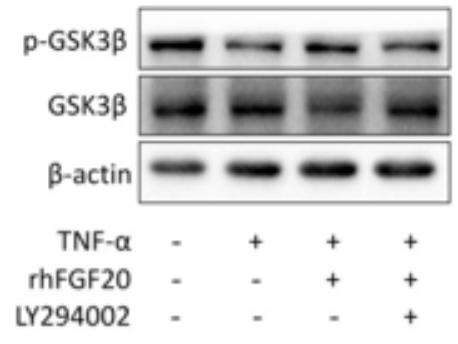

I

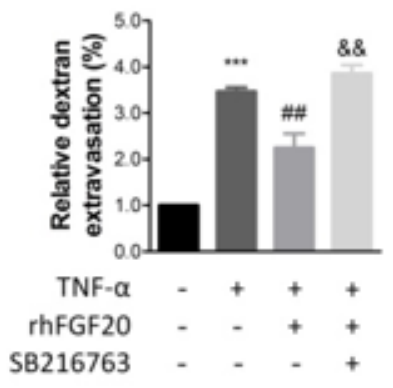

H

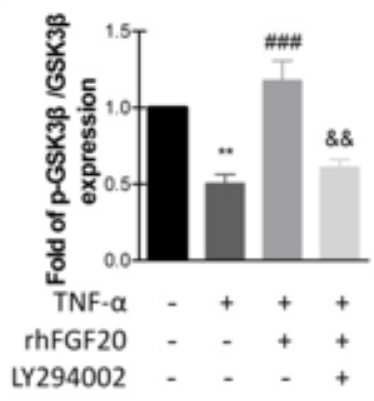

J

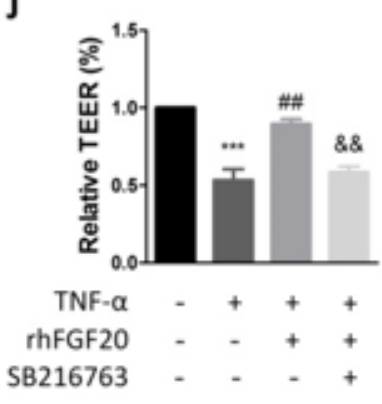

\section{Figure 8}

rhFGF20 ameliorates HBMEC monolayer disruption through AKT/GSK3 $\beta$ activation. (A) Effects of rhFGF20 on p-AKT and AKT expression, as shown by western blot analysis of the total cellular protein, with $\beta$-actin used as the loading control and for band density normalization. (B) Effects of rhFGF20 on pAKT/AKT protein levels analyzed by quantified western blotting data. (C-D) The AKT inhibitor LY294002 $(20 \mu \mathrm{M})$ reverses rhFGF20-mediated rescue of changes to FITC-dextran transport and decreased endothelial resistance. (E-F) LY294002 blocks the effect of rhFGF20 on Claudin-5 expression, as shown by western blot analysis of the total cellular proteins, with $\beta$-actin used as the loading control and for band density normalization. (G, H) Effects of rhFGF20 and LY294002 on p-GSK3 $\beta$ and GSK3 $\beta$ expression, as shown by western blot analysis of the total cellular protein, with $\beta$-actin used as the loading control and for band density normalization. (I-J) The GSK3 $\beta$ inhibitor SB216763 (30 $\mu \mathrm{M})$ reverses rhFGF20mediated rescue of changes to FITC-dextran transport and decreased endothelial resistance. ${ }^{\star *} \mathrm{P}<0.01$, ***P $<0.001$ vs. control group. \#P $<0.05$, \#\#P $<0.01$, \#\#\#P $<0.001$ vs. TNF-a group. \&P $<0.05, \& \& P<$ 0.01 vs. TNF- $a+$ rhFGF20 group. Data are presented as the mean values \pm SEM $(n=5)$. 
A

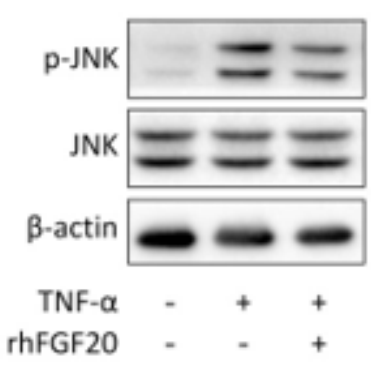

C

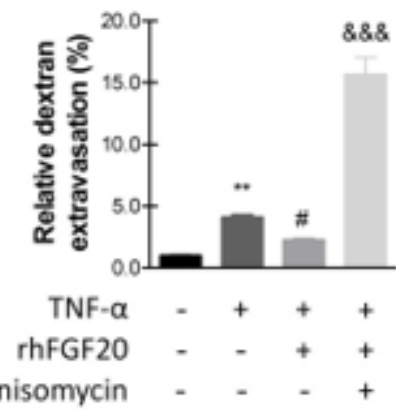

B

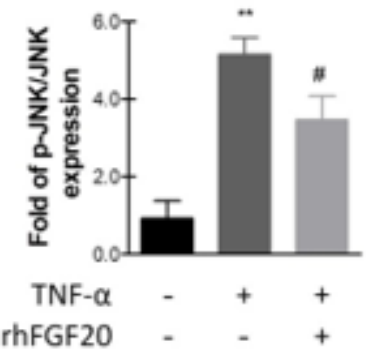

D

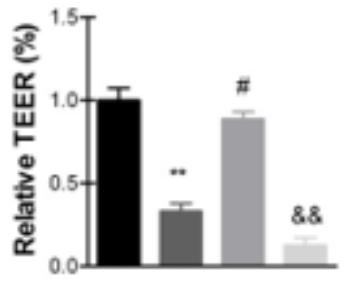

TNF- $\alpha-+++$

ThFGF20 - -++

Anisomycin - - - +
E

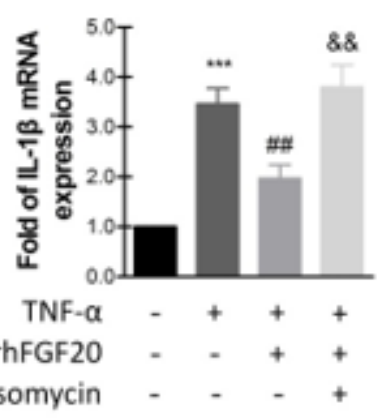

$\mathbf{F}$

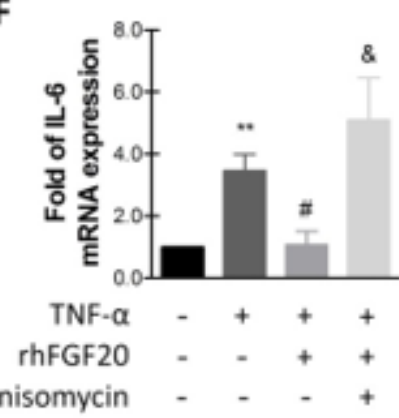

G

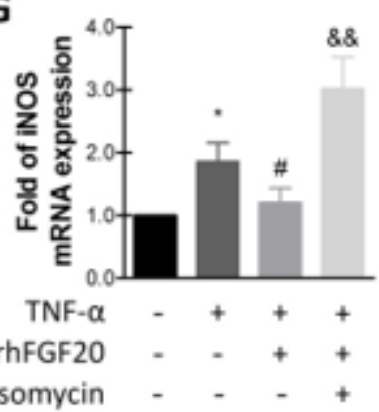

\section{Figure 9}

rhFGF20 ameliorates HBMEC monolayer disruption through the JNK/NFKB signaling pathway. (A) Effects of rhFGF20 on p-JNK and JNK expression, as shown by western blot analysis, with $\beta$-actin used as the loading control and for band density normalization. (B) Effects of rhFGF20 on p-AKT/AKT protein levels, as analyzed by quantified western blotting data. (C-D) The JNK inhibitor anisomycin $(20 \mu \mathrm{M})$ reverses the rhFGF20-mediated rescue of changes to FITC-dextran transport and decreased endothelial resistance. (E$\mathrm{G})$ Anisomycin blocks the effect of rhFGF20 on the mRNA levels of the proinflammatory cytokines IL-1 $\beta$, IL-6, and iNOS. ${ }^{*} P<0.05,{ }^{*} \mathrm{P}<0.05,{ }^{\star} * \star \mathrm{P}<0.001$ vs. control group. $\# \mathrm{P}<0.05, \# \# \mathrm{P}<0.01$ vs. TNF-a group. \&P $<0.05, \& \& P<0.01, \& \& \& P<0.001$ vs. TNF-a + rhFGF20 group. Data are presented as the mean values $\pm \operatorname{SEM}(n=5)$. 
A

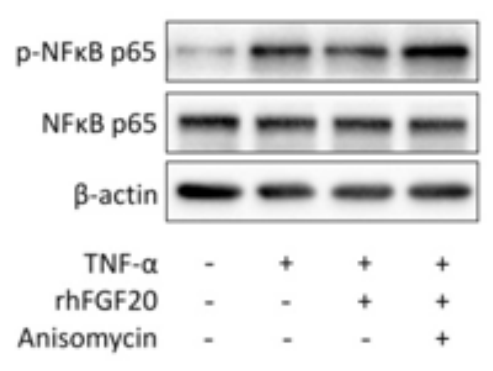

B

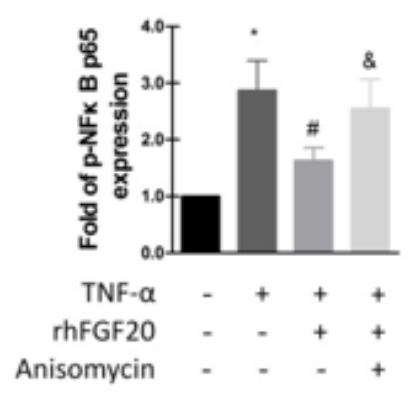

C
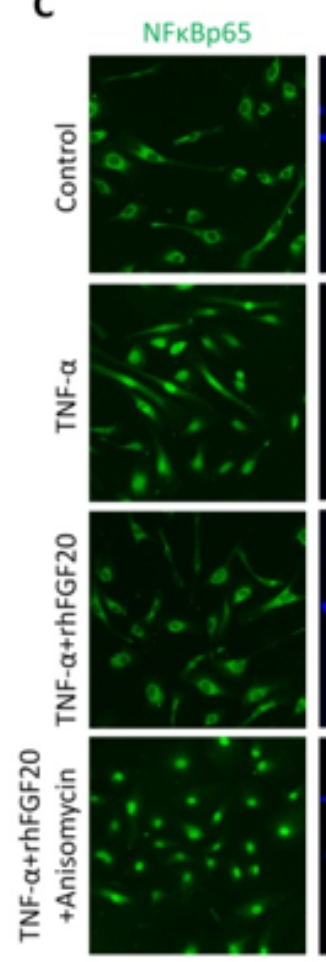

Dapi
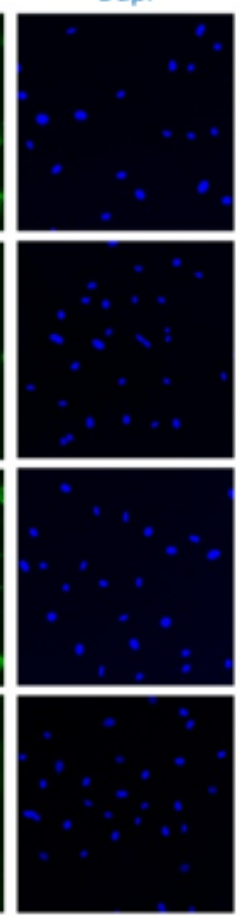

Merge
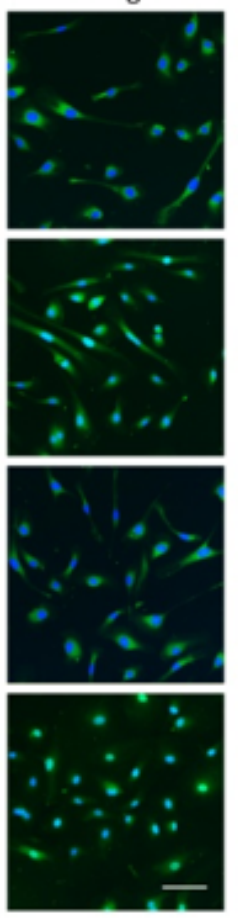

D

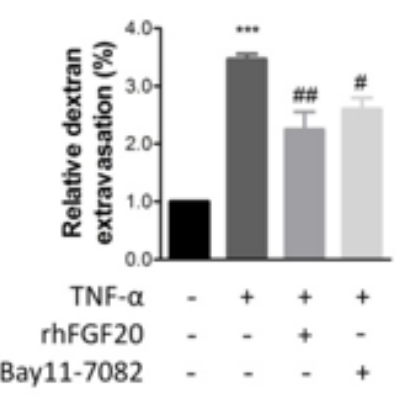

E

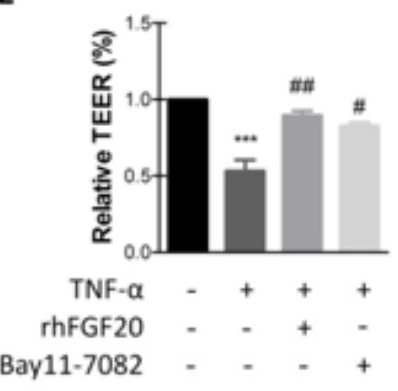

Figure 10

rhFGF20 ameliorates HBMEC monolayer disruption through JNK/NFKB activation. (A) Effects of rhFGF20 and anisomycin on p-NFKB p65 and NFKB p65 expression, as shown by western blot analysis of the total cellular protein, with $\beta$-actin used as the loading control and for band density normalization. (B) Effects of rhFGF20 and anisomycin on p-NFKB p65/NFKB p65 protein levels, as analyzed by quantified western blotting data. (C) Effects of rhFGF20 and anisomycin on NFKB p65 activity. Nuclei are labeled with DAPI. (D-E) The NFKB inhibitor Bay 11-7082 reverses rhFGF20-mediated rescue of changes in FITC-dextran transport and decreased endothelial resistance. ${ }^{*} \mathrm{P}<0.05$, ${ }^{\star \star *} \mathrm{P}<0.01$ vs. control group. \#P $<0.05, \# \# \mathrm{P}<$ 0.01 vs. TNF-a group. \&P $<0.05$ vs. TNF- $a+$ rhFGF20 group. Data are presented as the mean values $\pm \operatorname{SEM}(n=5)$. 


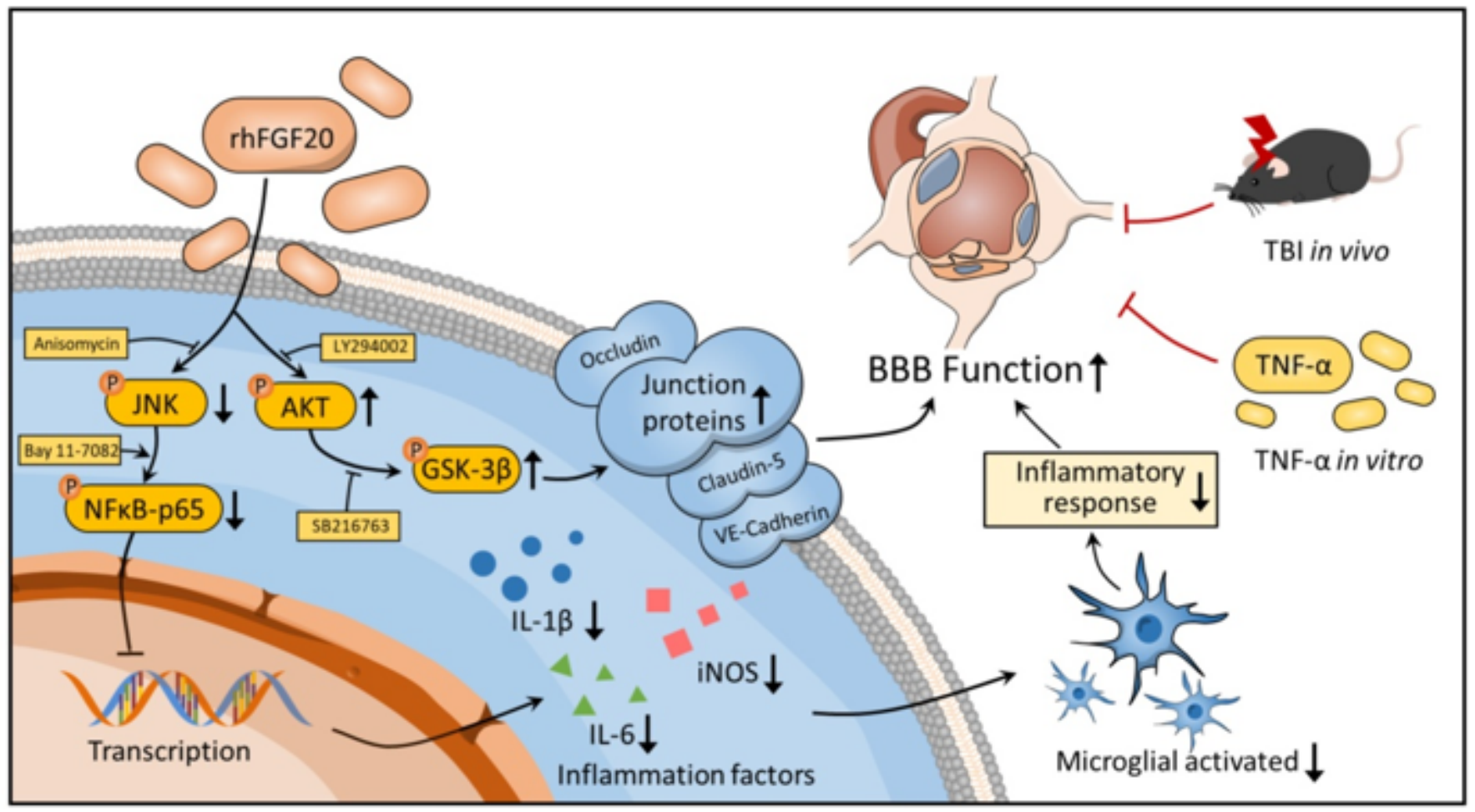

\section{Figure 11}

Potential signaling pathways involved in rhFGF20-mediated protection against BBB disruption in both in vivo TBI mice and an in vitro TNF-a-induced HBMEC model. rhFGF20 upregulates junction proteins via the AKT/GSK3 $\beta$ pathway and inhibits microglial activation and proinflammatory cytokine expression by suppressing the JNK/NFKB pathway, eventually protecting against BBB disruption. 